\title{
LONG-TIME ASYMPTOTICS FOR THE NONLOCAL MKDV EQUATION
}

\author{
FENGJING HE${ }^{1}$, ENGUI FAN ${ }^{1}$ AND JIAN XU² \\ 1. School of Mathematical Sciences, Fudan University, Shanghai 200433, P.R. China. \\ 2. College of Science, University of Shanghai for Science and Technology, Shanghai 200093, P. \\ R. China
}

ABstraCt. In this paper, we study the Cauchy problem with decaying initial data for the nonlocal modified Korteweg-de Vries equation (nonlocal mKdV)

$$
q_{t}(x, t)+q_{x x x}(x, t)-6 q(x, t) q(-x,-t) q_{x}(x, t)=0,
$$

which can be viewed as a generalization of the local classical mKdV equation. We first formulate the Riemann-Hilbert problem associated with the Cauchy problem of the nonlocal mKdV equation. Then we apply the Deift-Zhou nonlinear steepest-descent method to analyze the long-time asymptotics for the solution of the nonlocal $\mathrm{mKdV}$ equation. In contrast with the classical $\mathrm{mKdV}$ equation, we find some new and different results on long-time asymptotics for the nonlocal mKdV equation and some additional assumptions about the scattering data are made in our main results.

AMS Subject Classification (2010): 41A60, 35Q15, 35Q53.

KEYwords: nonlocal mKdV equation, Riemann-Hilbert problem, nonlinear steepest-descent, long-time asymptotics.

\section{Contents}

1. Introduction 2

2. Inverse scattering transform and the Riemann-Hilbert problem 4

3. Reduction to a model RH problem 7

4. Long time asymptotics 15

Appendix A. 25

References 25

E-mail address: fjhe16@fudan.edu.cn; faneg@fudan.edu.cn; jianxu@usst.edu.cn. 


\section{INTRODUCTION}

The pioneering work for the nonlocal systems has been done by Ablowitz and Musslimani when they studied the nonlocal NLS equation with PT symmetry [3]. This research field has attracted much attention from both mathematics and the physical application of nonlinear optics and magnetics [14, 24, 25]. Since the nonlocal NLS was found, a number of other nonlocal integrable systems has been introduced from a mathematical viewpoint. For instance, from symmetry reduction of general AKNS system, some new reverse space-time and reverse time nonlocal nonlinear integrable version of the NLS, $\mathrm{mKdV}$, sine-Gordon equation were found [5]. Recently, Yang constructed some new nonlocal integrable equations by simple variable transformations on local equations [32.

Like the local case, the nonlocal integrable systems also possess integrable properties, for example, nonlocal NLS equation admits infinite number of conservation laws and can be solved by using the inverse scattering transform (IST) [4]. Some exact solutions of nonlocal $\mathrm{mKdV}$ equation including soliton, kink, rogue-wave and breather were obtained through either Darboux transformation or IST. These solutions have displayed some new properties which are different from those of local equation [18, 19]. In physical application, the nonlocal mKdV possesses the shifted parity and delayed time reveal symmetry, and thus it can be related to the Alice-Bob system[23. For instance, a special approximate solution of the nonlocal $\mathrm{mKdV}$ was applied to theoretically capture the salient features of two correlated dipole blocking events in atmospheric dynamical systems [27].

However, there has been still not much work on the Riemann-Hilbert method to the nonlocal systems except to the recent paper [26], where Rybalko and Shepelsky obtained the long-time asymptotics of the solution for the nonlocal Schrodinger equation via the nonlinear steepest-descent method. In this paper, we apply Riemann-Hilbert (RH) method and Deift-Zhou nonlinear steepest-descent method to analyze longtime asymptotics of the Cauchy problem of the nonlocal mKdV equation

$$
\begin{aligned}
& q_{t}(x, t)+q_{x x x}(x, t)-6 q(x, t) r(x, t) q_{x}(x, t)=0, \\
& q(x, 0)=q_{0}(x)
\end{aligned}
$$

where $r(x, t)=q(-x,-t)$ is a symmetry reduction of an AKNS system, and the initial data $q_{0}(x)$ decays rapidly to zero as $x \rightarrow \pm \infty$.

In 1970's, the solutions of the Cauchy problem for many integrable nonlinear wave equations was obtained by solving an associated $\mathrm{RH}$ problem on the complex plane [2]. More precisely, starting with initial data, the direct scattering transform gives rise to certain spectral functions whose time evolution is simple. Then the solution of the original 
Cauchy problem can be recovered via the IST characterized in terms of $\mathrm{RH}$ problem whose jump matrix depends on the given spectral functions.

In 1993, Deift and Zhou introduced the nonlinear steepest-descent method to analyze the asymptotics of the solutions of $\mathrm{RH}$ problems [12]. It involves a series of counter deformation aiming to reduce the original $\mathrm{RH}$ problem to the one whose jump matrix is decaying fast (as $t \rightarrow \infty$ ) to the identity matrix everywhere except near some stationary phase points; and it is the contour near these points that determine the leading order of the long time asymptotics which can be obtained explicitly after rescaling the RH problem. This method has been used to study rigorously the long-time asymptotics of a wide variety of integral systems, such as the mKdV equation [12] and the non-focusing NLS equation [11, the sine-Gordon equation [10], the modified Schrödinger equation [20, 21], the KdV equation [15], the Cammasa-Holm equation [9], Fokas-Lenells equation[29], derivative NLS equation [31], short pulse equation [28, 30], Sine-Gordon equation [16], Kundu-Eckhaus Equation[33].

In [12, Deift and Zhou obtained the explicit leading order long-time asymptotic behavior of the solution to the classical mKdV equation

$$
\begin{aligned}
& q_{t}(x, t)+q_{x x x}(x, t)-6 q^{2}(x, t) q(x, t)=0, \\
& q(x, 0)=q_{0}(x)
\end{aligned}
$$

using the nonlinear steepest descent method. Here we extend above results to give the asymptotic behavior of solution of nonlocal mKdV equation (1.1), but it will be much different from that on the classical mKdV equation 1.2 in the following three aspects.

(i) For our nonlocal mKdV equation, the jump matrix of the $\mathrm{RH}$ problem involve two reflection coefficients $r_{1}(k)$ and $r_{2}(k)$, but there is only one reflection coefficient $r(k)$ for the local mKdV equation, which is specified by $r_{1}(k)=r(k), \quad r_{2}(k)=\overline{r(\bar{k})},|r(k)|<1$.

(ii) In the analysis of the local equations, the great difference from the nonlocal case is that $1-r_{1}(k) r_{1}(k)$ is complex-valued, which leads to $\operatorname{Im} \nu(\zeta) \neq 0$. We will find below that $\operatorname{Im} \nu(\zeta)$ contributes to both the leading order and the error terms in the asymptotics for the nonlocal $\mathrm{mKdV}$ equation. To obtain asymptotic behavior of solution of nonlocal mKdV equation, we have used Slightly different method from that in [12].

(iii) At last, in contrast with the asymptotic of local $\mathrm{mKdV}$ equation, we obtain the long time asymptotic of nonlocal $\mathrm{mKdV}$ equation as follows

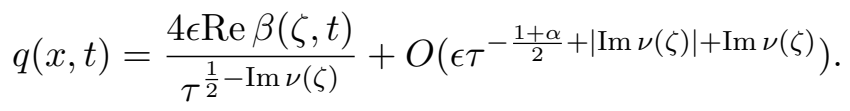

Note that the decay rate of the leading term depends on $\zeta=\frac{x}{t}$ through $\operatorname{Im} \nu(\zeta)$, while $\operatorname{Im} \nu(\zeta)=0$ for all $\zeta \in \mathcal{I}$ in the local $\mathrm{mKdV}$ equation. 
Organization of this paper is as follows. In Section 2, we present the IST and express the solution of nonlocal mKdV equation (1.1) in terms of a $\mathrm{RH}$ problem. In Section 3, we conduct several deformations to obtain a model $\mathrm{RH}$ problem convenient for consequent analysis. In Section 4, we derive the long-time behavior of nonlocal mKdV equation (1.1) in the similarity sector.

\section{Inverse SCATtering tRAnsform And the Riemann-Hilbert PRoblem}

Since 1.1a) is a member of AKNS systems, the standard method of IST was applied in [19]. We reformulate the IST to express the solution of (1.1) in terms of a RH problem for convenience of the consequent analysis.

The nonlocal mKdV equation 1.1a admits the Lax pair

$$
\begin{aligned}
& \Phi_{x}+i k \sigma_{3} \Phi=U \Phi, \\
& \Phi_{t}+4 i k^{3} \sigma_{3} \Phi=V \Phi,
\end{aligned}
$$

where $\Phi(x, t, k)$ is a $2 \times 2$-matrix valued eigenfunction, $k \in \mathbb{C}$ is the spectral parameter, and

$$
\sigma_{3}=\left(\begin{array}{cc}
1 & 0 \\
0 & -1
\end{array}\right), \quad U=\left(\begin{array}{cc}
0 & q(x, t) \\
q(-x,-t) & 0
\end{array}\right), \quad V=\left(\begin{array}{cc}
A & B \\
C & -A
\end{array}\right)
$$

with

$$
\begin{aligned}
& A=-2 i q(x, t) q(-x,-t) k+q(-x,-t) q_{x}(x, t)+q(x, t) q_{x}(-x,-t), \\
& B=4 k^{2} q(x, t)+2 i q_{x}(x, t) k+2 q^{2}(x, t) q(-x,-t)-q_{x x}(x, t), \\
& C=4 k^{2} q(-x,-t)+2 i q_{x}(-x,-t) k+2 q^{2}(x, t) q(-x,-t)-q_{x x}(-x,-t) .
\end{aligned}
$$

Let $\Psi_{j}(x, t, k), j=1,2$, be the $2 \times 2$-matrix valued solutions of the linear Volterra integral equations

$$
\begin{aligned}
& \Psi_{1}(x, t, k)=I+\int_{-\infty}^{x} e^{i k(y-x) \hat{\sigma}_{3}}\left(U(y, t) \Psi_{1}(y, t, k)\right) d y, \quad k \in\left(\mathbb{C}_{+}, \mathbb{C}_{-}\right), \\
& \Psi_{2}(x, t, k)=I+\int_{\infty}^{x} e^{i k(y-x) \hat{\sigma}_{3}}\left(U(y, t) \Psi_{2}(y, t, k)\right) d y, \quad k \in\left(\mathbb{C}_{-}, \mathbb{C}_{+}\right)
\end{aligned}
$$

where $\hat{\sigma}_{3}$ acts on a $2 \times 2$ matrix $A$ by $\hat{\sigma}_{3} A=\left[\hat{\sigma}_{3}, A\right]$, i.e. $e^{\hat{\sigma}_{3}} A=e^{\sigma_{3}} A e^{-\sigma_{3}}, \mathbb{C}_{ \pm}=$ $\{k \in \mathbb{C} \mid \pm \operatorname{Im} k>0\}$, and the notation $k \in\left(\mathbb{C}_{+}, \mathbb{C}_{-}\right)$indicates that the first and second columns are valid for $k \in \mathbb{C}_{+}$and $k \in \mathbb{C}_{-}$, respectively. From $(2.2)$, we can prove that $\Psi_{1}(x, t, \cdot)$ is continuous for $k \in\left(\overline{\mathbb{C}_{+}}, \overline{\mathbb{C}_{-}}\right)$and analytic for $k \in\left(\mathbb{C}_{+}, \mathbb{C}_{-}\right), \Psi_{2}(x, t, \cdot)$ is continuous for $k \in\left(\overline{\mathbb{C}_{-}}, \overline{\mathbb{C}_{+}}\right)$and analytic for $k \in\left(\mathbb{C}_{-}, \mathbb{C}_{+}\right)$[7]. Moreover we can derive the large $k$ asymptotics of $\Psi_{j}$ (c.f. [7])

$$
\Psi_{j}(x, t, k)=I+O\left(k^{-1}\right), \quad k \rightarrow \infty,
$$

where the error term is uniformly with respect to $x, t$. 
Then the Jost solutions $\Phi_{j}(x, t, k), j=1,2$, of $(2.1)$ are defined as follow

$$
\Phi_{j}(x, t, k)=\Psi_{j}(x, t, k) e^{\left(-i k x-4 i k^{3} t\right) \sigma_{3}} .
$$

Since $U$ is traceless, $\operatorname{det} \Phi_{j}(x, t, k) \equiv 1$ for all $x, t$, and $k$. And for $k \in \mathbb{R}, \Phi_{j}(x, t, k)$ can be related by scattering matrix $S(k)$

$$
\Phi_{1}(x, t, k)=\Phi_{2}(x, t, k) S(k), \quad k \in \mathbb{R},
$$

where

$$
S(k)=\left(\begin{array}{ll}
s_{11}(k) & s_{12}(k) \\
s_{21}(k) & s_{22}(k)
\end{array}\right), \quad k \in \mathbb{R},
$$

is independent of $x$ and $t$.

We now establish important symmetry properties of the scattering matrix (2.6). It can be verified that if $\Psi(x, t, k)$ is the solution of $2.2 \mathrm{a})$, then $\Lambda \overline{\Psi(-x,-t,-\bar{k})} \Lambda^{-1}$ is the solution of $2.2 \mathrm{~b}$ with $\Lambda=\left(\begin{array}{cc}0 & -1 \\ 1 & 0\end{array}\right)$. Notice 2.4 and the uniqueness of the solution of the Volterra equation 2.2 , we arrives that

$$
\Phi_{2}^{(2)}(x, t, k)=\Lambda \overline{\Phi_{1}^{(1)}(-x,-t,-\bar{k})}, \quad \Phi_{2}^{(1)}(x, t, k)=\Lambda^{-1} \overline{\Phi_{1}^{(2)}(-x,-t,-\bar{k})},
$$

where $\Phi_{i}^{(j)}(x, t, k)$ denotes the $j$-th column of the matrix $\Phi_{i}(x, t, k)$. Rewrite the relation between the Jost solutions (2.5) as

$$
\begin{aligned}
& \Phi_{1}^{(1)}(x, t, k)=s_{11}(k) \Phi_{2}^{(1)}(x, t, k)+s_{21}(k) \Phi_{2}^{(2)}(x, t, k), \\
& \Phi_{1}^{(2)}(x, t, k)=s_{12}(k) \Phi_{2}^{(1)}(x, t, k)+s_{22}(k) \Phi_{2}^{(2)}(x, t, k),
\end{aligned}
$$

the scattering data can be represented in terms of $\Phi_{i}^{(j)}$, and from 2.7), we reach the following symmetry

$$
\begin{aligned}
s_{11}(k) & =\operatorname{det}\left(\Phi_{1}^{(1)}(x, t, k), \Phi_{2}^{(2)}(x, t, k)\right) \\
& =\operatorname{det}\left(\Lambda^{-1}\left(\overline{\Phi_{1}^{(1)}(-x,-t,-\bar{k})}, \overline{\Phi_{2}^{(2)}(-x,-t,-\bar{k})}\right) \Lambda\right)=\overline{s_{11}(-\bar{k})}, \\
s_{22}(k) & =\operatorname{det}\left(\Phi_{2}^{(1)}(x, t, k), \Phi_{1}^{(2)}(x, t, k)\right) \\
& =\operatorname{det}\left(\Lambda^{-1}\left(\overline{\Phi_{2}^{(1)}(-x,-t,-\bar{k})}, \overline{\Phi_{1}^{(2)}(-x,-t,-\bar{k})}\right) \Lambda\right)=\overline{s_{22}(-\bar{k})}, \\
s_{12}(k) & =\operatorname{det}\left(\Phi_{1}^{(2)}(x, t, k), \Phi_{2}^{(2)}(x, t, k)\right) \\
& =\operatorname{det}\left(\Lambda\left(\overline{\Phi_{2}^{(1)}(-x,-t,-\bar{k})}, \overline{\Phi_{1}^{(1)}(-x,-t,-\bar{k})}\right)\right)=\overline{s_{21}(-\bar{k})} .
\end{aligned}
$$

Further more, we can also verify that if $\Psi(x, t, k)$ is the solution of $(2.2 \mathrm{a})$, then $\Lambda \Psi(-x,-t, k) \Lambda^{-1}$ is the solution of $(2.2 \mathrm{~b})$. So following the above procedure, we obtain another symmetry property

$$
s_{12}(k)=s_{21}(k) .
$$

Finally, from $(2.9)$ and 2.10$), S(k)$ can be written in the form

$$
S(k)=\left(\begin{array}{cc}
a_{1}(k) & b(k) \\
b(k) & a_{2}(k)
\end{array}\right),
$$


where

$$
a_{1}(k)=\overline{a_{1}(-\bar{k})}, \quad a_{2}(k)=\overline{a_{2}(-\bar{k})}, \quad b(k)=\overline{b(-\bar{k})} .
$$

In accordance with the case of local equations [7, 17], the scattering matrix $S(k)$ is uniquely determined by the initial data $q_{0}(x)$, and we can conclude that:

(1) $a_{1}(k)$ is analytic for $k \in \mathbb{C}_{+}$, and continuous for $k \in \overline{\mathbb{C}_{+}} ; a_{2}(k)$ is analytic for $k \in \mathbb{C}_{-}$, and continuous for $k \in \overline{\mathbb{C}_{-}}$.

(2) $a_{j}(k)=1+O\left(k^{-1}\right), j=1,2$ and $b(k)=O\left(k^{-1}\right)$ as $k \rightarrow \infty$.

(3) $a_{1}(k)=\overline{a_{1}(-\bar{k})}, k \in \overline{\mathbb{C}_{+}} ; \quad a_{2}(k)=\overline{a_{2}(-\bar{k})}, k \in \overline{\mathbb{C}_{-}} ; \quad b(k)=\overline{b(-\bar{k})}, k \in \mathbb{R}$.

(4) $a_{1}(k) a_{2}(k)-b(k)^{2}=1, k \in \mathbb{R}$, (follows from $\operatorname{det} S(k)=1$ ).

Now we define the matrix valued function $M$ as

$$
M(x, t, k)=\left\{\begin{array}{l}
\left(\frac{\Psi_{1}^{(1)}(x, t, k)}{a_{1}(k)}, \Psi_{2}^{(2)}(x, t, k)\right), \operatorname{Im} k>0, \\
\left.\Psi_{2}^{(1)}(x, t, k), \frac{\Psi_{1}^{(2)}(x, t, k)}{a_{2}(k)}\right), \operatorname{Im} k<0 .
\end{array}\right.
$$

Using scattering relation (2.5), we have the jump condition for $M(x, t, k)$ across $k \in \mathbb{R}$

$$
M_{+}(x, t, k)=M_{-}(x, t, k) J(x, t, k), \quad k \in \mathbb{R},
$$

where $M_{ \pm}$is the limiting value of $M$ as $k$ approaches $\mathbb{R}$ from $\mathbb{C}_{ \pm}$, and

$$
J(x, t, k)=e^{\left(-i k x-4 i k^{3} t\right) \hat{\sigma}_{3}}\left(\begin{array}{cc}
1-r_{1}(k) r_{2}(k) & -r_{2}(k) \\
r_{1}(k) & 1
\end{array}\right), \quad k \in \mathbb{R},
$$

and reflection coefficients are defined by

$$
r_{1}(k)=\frac{b(k)}{a_{1}(k)}, \quad r_{2}(k)=\frac{b(k)}{a_{2}(k)} .
$$

From the symmetry of scattering data 2.12,,$r_{1}$ and $r_{2}$ also possess the symmetry property

$$
r_{1}(k)=\overline{r_{1}(-\bar{k})}, \quad r_{2}(k)=\overline{r_{2}(-\bar{k})}, \quad k \in \mathbb{R},
$$

and the determinant property 4 implies that

$$
1-r_{1}(k) r_{2}(k)=\frac{1}{a_{1}(k) a_{2}(k)}, \quad k \in \mathbb{R} .
$$

We assume that $a_{1}(k)$ and $a_{2}(k)$ have no zeros in $\overline{\mathbb{C}_{+}}$and $\overline{\mathbb{C}_{-}}$respectively so that one can assemble the above facts into the form of a Riemann-Hilbert problem

$$
\left\{\begin{array}{l}
M(x, t, k) \text { analytic in } \mathbb{C} \backslash \mathbb{R}, \\
M_{+}(x, t, k)=M_{-}(x, t, k) J(x, t, k), \quad k \in \mathbb{R}, \\
M(x, t, k) \rightarrow I, \quad k \rightarrow \infty
\end{array}\right.
$$

Remark 2.1. $R H$ problem (2.19) can be regard as a generalization of the $R H$ problem associated with the $m K d V$ equation. In the local case, the reflection coefficients are specified by

$$
r_{1}(k)=r(k), \quad r_{2}(k)=\overline{r(\bar{k})}, \quad k \in \mathbb{R},
$$

with $|r(k)|<1$. 
Inversely, if $\mathrm{RH}$ problem 2.19 has a unique solution for all $(x, t)$, the solution $q(x, t)$ of (1.1) is given by (c.f. [1, 8 , 13])

$$
q(x, t)=2 i \lim _{k \rightarrow \infty}(k M(x, t, k)) 12 .
$$

\section{REDUCtion to A MODEL RH PROBLEM}

The deformations of the $\mathrm{RH}$ problem (2.19) are similar to the local case [12, 22], where the original $\mathrm{RH}$ problem is deformed to the one whose jump matrix decays to the identity matrix everywhere as $t \rightarrow \infty$ except near the stationary points. Then an explicitly solvable $\mathrm{RH}$ problem is introduced to obtain a model $\mathrm{RH}$ problem for which long time asymptotics can be conveniently performed.

Let $\mathcal{I}=[-N, 0)$ be the interval with $N>0$ and let $\zeta=x / t$ be the variable with $\zeta \in \mathcal{I}$. Let $M(x, t, \cdot)$ denote the unique solution of the $\mathrm{RH}$ problem 2.19 , and the phase of the exponentials $e^{ \pm t \Phi(\zeta)}$ in the jump matrix 2.15 is defined by

$$
\Phi(\zeta, k)=2 i k \zeta+8 i k^{3}
$$

which admits two stationary points

$$
k_{0}=\sqrt{-\frac{\zeta}{12}}, \quad k_{0}=-\sqrt{-\frac{\zeta}{12}}
$$

such that $\frac{d \Phi}{d k}\left( \pm k_{0}\right)=0$.

Now we deform the RH problem 2.19 with the following steps.

Step 1 The first step is to search for upper/lower and lower/upper triangular factorizations of the jump matrix. For this purpose, we introduce a scalar RH problem

$$
\left\{\begin{array}{l}
\delta \text { analytic in } \mathbb{C} \backslash\left[-k_{0}, k_{0}\right], \\
\delta_{+}=\delta_{-}\left(1-r_{1}(k) r_{2}(k)\right), \quad|k|<k_{0}, \\
\delta \rightarrow 1 \quad k \rightarrow \infty
\end{array}\right.
$$

Direct calculation shows that (3.3) admits a unique solution

$$
\delta(\zeta, k)=e^{\frac{1}{2 \pi i} \int_{-k_{0}}^{k_{0}} \ln \left(1-r_{1}(s) r_{2}(s)\right) \frac{d s}{s-k}}, \quad k \in \mathbb{C} \backslash\left[-k_{0}, k_{0}\right] .
$$

The symmetry (2.17) implies that

$$
\delta(\zeta, k)=\overline{\delta(\zeta,-\bar{k})}
$$

moreover, integrating by parts in formula (3.4) yields

$$
\delta(\zeta, k)=\frac{\left(k-k_{0}\right)^{i \nu(\zeta)}}{\left(k+k_{0}\right)^{i \nu(\zeta)}} e^{\tilde{\chi}(\zeta, k)},
$$

where $\tilde{\chi}(\zeta, k)$ is a uniformly bounded function with respect to $\zeta \in \mathcal{I}$ and $k \in \mathbb{C} \backslash \mathbb{R}$, which is defined by

$$
\tilde{\chi}(\zeta, k)=-\frac{1}{2 \pi i} \int_{-k_{0}}^{k_{0}} \ln (k-s) d \ln \left(1-r_{1}(s) r_{2}(s)\right),
$$


and $\nu(\zeta)$ is a bounded function defined by

$$
\nu(\zeta)=-\frac{1}{2 \pi} \ln \left(1-r_{1}\left(k_{0}\right) r_{2}\left(k_{0}\right)\right)=-\frac{1}{2 \pi} \ln \left|1-r_{1}\left(k_{0}\right) r_{2}\left(k_{0}\right)\right|-\frac{i}{2 \pi} \Delta(\zeta)
$$

with

We assume that

$$
\Delta(\zeta)=\int_{-\infty}^{k_{0}} d \arg \left(1-r_{1}(s) r_{2}(s)\right)
$$

$$
\Delta(\zeta) \in(-\pi, \pi), \quad \zeta \in \mathcal{I},
$$

then $\nu(\zeta)$ is single valued and

$$
|\operatorname{Im} \nu(\zeta)|<\frac{1}{2}, \quad \zeta \in \mathcal{I}
$$

Consequently the singularity of $\delta(\zeta, k)$ at $k= \pm k_{0}$ is square integrable.

$\delta(\zeta, k)$ can be written in another way:

$$
\delta(\zeta, k)=\left(\frac{k-k_{0}}{k+k_{0}}\right)^{i \nu(\zeta)} e^{\chi(\zeta, k)},
$$

where

$$
\begin{aligned}
\chi(\zeta, k) & =\frac{1}{2 \pi i} \int_{-k_{0}}^{k_{0}} \ln \left(\frac{1-r_{1}(s) r_{2}(s)}{1-r_{1}\left(k_{0}\right) r_{2}\left(k_{0}\right)}\right) \frac{d s}{s-k} \\
& =\tilde{\chi}(\zeta, k)-\frac{1}{2 \pi i} \ln \left(\frac{1-\overline{r_{1}\left(k_{0}\right) r_{2}\left(k_{0}\right)}}{1-r_{1}\left(k_{0}\right) r_{2}\left(k_{0}\right)}\right) \ln \left(k+k_{0}\right) .
\end{aligned}
$$

In the local case, $\chi(\zeta, k)$ is equivalent to $\tilde{\chi}(\zeta, k)$ by symmetry 2.20 , so $\chi(\zeta, k)$ is uniformly bounded. However $\chi(\zeta, k)$ is singular at $k=-k_{0}$ for nonlocal equation.

Lemma 3.1. Let $S=\left\{k^{\prime} \in \mathbb{C}|| k^{\prime}+k_{0} \mid \geq \frac{k_{0}}{2}\right\}$ denote the complex plane minusing a neighborhood of $-k_{0}$. Then $\chi(\zeta, k)$ is uniformly bounded with respect to $\zeta \in \mathcal{I}$ and $k \in S$, i.e.

$$
\sup _{\zeta \in \mathcal{I}} \sup _{k \in S}|\chi(\zeta, k)| \leq C
$$

Proof. Since $\delta(\zeta, k) \rightarrow 1$ as $k \rightarrow \infty, \chi(\zeta, k)$ is uniformly bounded with respect to $\zeta \in \mathcal{I}$ and $k \in\left\{k^{\prime} \in \mathbb{C}|| k^{\prime}+k_{0} \mid \geq G\right\}$ by (3.11), where $G$ is a large enough constant. Let $k=-k_{0}+u e^{i \alpha}$, where $\frac{k_{0}}{2} \leq u<G$ and $\alpha \in(-\pi, \pi]$. By 3.12

$$
\begin{aligned}
|\chi(\zeta, k)| & \leq C+C\left|\ln \left(\frac{1-\overline{r_{1}\left(k_{0}\right) r_{2}\left(k_{0}\right)}}{1-r_{1}\left(k_{0}\right) r_{2}\left(k_{0}\right)}\right)\right| \ln \left(u e^{i \alpha}\right) \mid \\
& \leq C+C\left|\ln \left(\frac{1-\overline{r_{1}\left(k_{0}\right) r_{2}\left(k_{0}\right)}}{1-r_{1}\left(k_{0}\right) r_{2}\left(k_{0}\right)}\right)\right|\left|\ln \frac{k_{0}}{2}\right| .
\end{aligned}
$$

Symmetry (2.17) implies that

$$
r_{j}(0)=\overline{r_{j}(0)}, \quad j=1,2,
$$

thus $\chi(\zeta, k)$ is also uniformly bounded with respect to $\zeta \in \mathcal{I}$ and $k \in\left\{k^{\prime} \in \mathbb{C} \mid \frac{k_{0}}{2} \leq\right.$ $\left.\left|k^{\prime}+k_{0}\right|<G\right\}$ by 3.14 and 3.15 


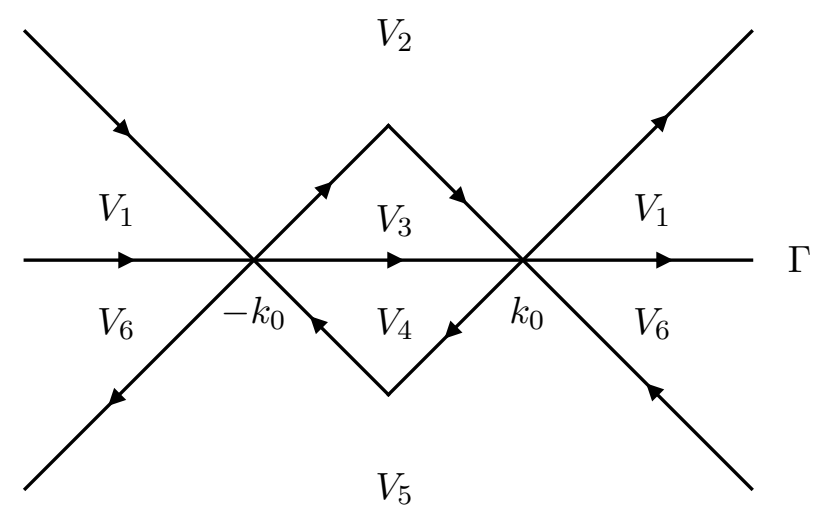

Figure 1. The jump contour $\Gamma$ and the open sets $\left\{V_{j}\right\}_{1}^{6}$

Remark 3.2. In the analysis of the local equations, the chief difference from the nonlocal case is that $1-r_{1}(k) r_{2}(k)$ is complex-valued, that is, $\operatorname{Im} \nu(\zeta) \neq 0$. We will find below that $\operatorname{Im} \nu(\zeta)$ contributes to both the leading order and the error terms in the asymptotics for the nonlocal $m K d V$ equation.

Conjugating the $\mathrm{RH}$ problem 2.19) by

$$
\delta(\zeta, k)^{-\sigma_{3}}=\left(\begin{array}{cc}
\delta(\zeta, k)^{-1} & 0 \\
0 & \delta(\zeta, k)
\end{array}\right)
$$

leads to the factorization problem for $\tilde{M}(x, t, k)=M(x, t, k) \delta(\zeta, k)^{-\sigma_{3}}$,

$$
\left\{\begin{array}{l}
\tilde{M}_{+}(x, t, k)=\tilde{M}_{-}(x, t, k) \tilde{J}(x, t, k), \quad k \in \mathbb{R}, \\
\tilde{M}(x, t, k) \rightarrow I, \quad k \rightarrow \infty
\end{array}\right.
$$

where

$$
\tilde{J}=\left\{\begin{array}{cc}
\left(\begin{array}{cc}
1 & -\delta(\zeta, k)^{2} r_{2}(k) e^{-t \Phi(\zeta, k)} \\
0 & 1
\end{array}\right)\left(\begin{array}{cc}
1 & 0 \\
\delta(\zeta, k)^{-2} r_{1}(k) e^{t \Phi(\zeta, k)} & 1
\end{array}\right), & |k|>k_{0}, \\
1 & 0 \\
\delta_{-}(\zeta, k)^{-2} r_{3}(k) e^{t \Phi(\zeta, k)} & 1
\end{array}\right)\left(\begin{array}{cc}
1 & -\delta_{+}(\zeta, k)^{2} r_{4}(k) e^{-t \Phi(\zeta, k)} \\
0 & 1
\end{array}\right), \quad|k|<k_{0}, \quad k \in \mathbb{R},
$$

with

$$
r_{3}(k)=\frac{r_{1}(k)}{1-r_{1}(k) r_{2}(k)}, \quad r_{4}(k)=\frac{r_{2}(k)}{1-r_{1}(k) r_{2}(k)} .
$$

Step 2 In accordance with the local case, we introduce oriented counter $\Gamma$ and open sets $\left\{V_{j}\right\}_{1}^{6}$ as depicted in Figure 1, and define $m(x, t, k)$ by 


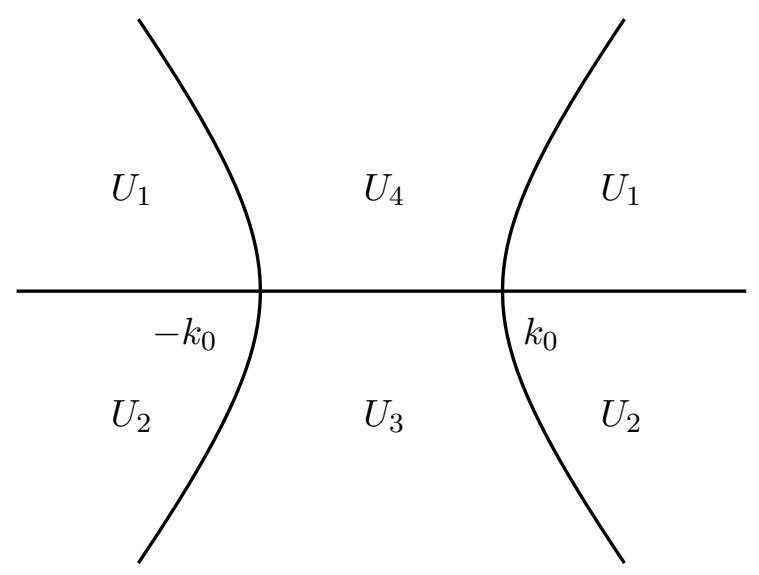

Figure 2. The domains $\left\{U_{j}\right\}_{1}^{4}$ in the complex $k$-plane. $\operatorname{Re} \Phi=0$ on the curves.

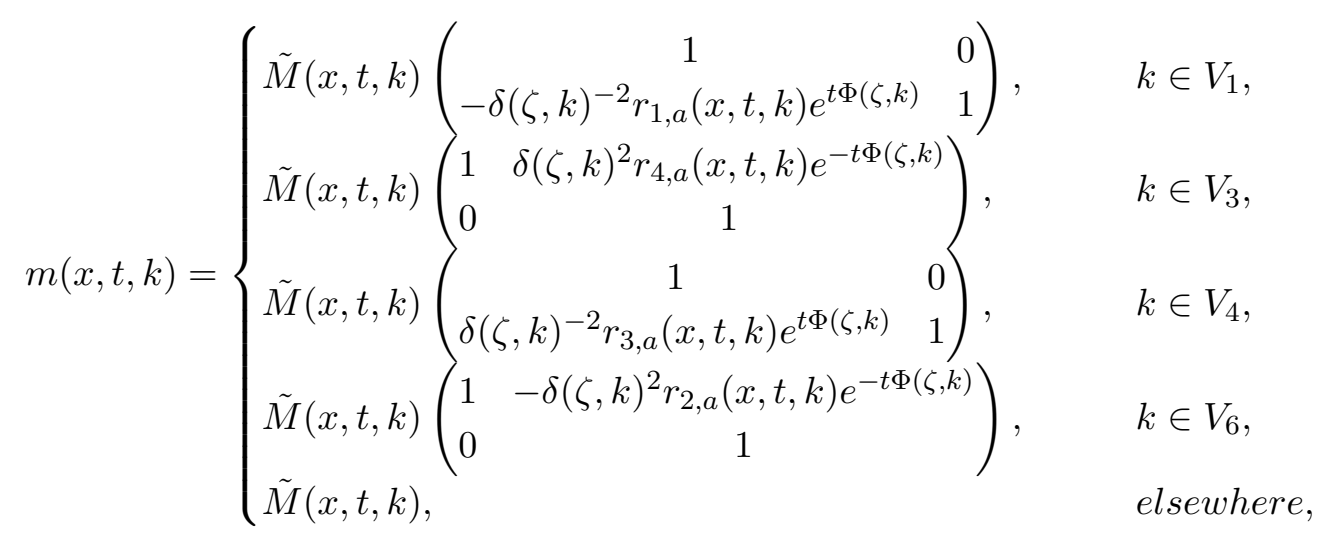

where $r_{j, a}$ is the analytic approximation of $r_{j}$ with small error $r_{j, r}, j=1, \cdots, 4$. More precisely, since $\left\{r_{j}(k)\right\}_{1}^{4}$ are sufficiently smooth and decaying, we can closely follow the proof of Lemma 4.8 in [22] to obtain similar decompositions:

Dividing the complex $k$-plane into four parts $U_{j}, j=1, \cdots, 4$ as in Figure 2 so that

$$
\{k \mid \operatorname{Re} \Phi(\zeta, k)<0\}=U_{1} \cup U_{3}, \quad\{k \mid \operatorname{Re} \Phi(\zeta, k)>0\}=U_{2} \cup U_{4},
$$

we can introduce decompositions

$$
r_{j}(k)= \begin{cases}r_{j, a}(x, t, k)+r_{j, r}(x, t, k), & j=1,2,|k|>k_{0}, k \in \mathbb{R}, \\ r_{j, a}(x, t, k)+r_{j, r}(x, t, k), & j=3,4,|k|<k_{0}, k \in \mathbb{R},\end{cases}
$$

such that 
(1) $r_{j, a}(x, t, k)$ is defined and continuous for $k \in \bar{U}_{j}$, analytic for $k \in U_{j}$, and for each $K>0$ satisfies

$$
\begin{aligned}
& \left|r_{j, a}(x, t, k)-r_{j}\left(k_{0}\right)\right| \leq C_{K}\left|k-k_{0}\right| e^{\frac{t}{4}|\operatorname{Re} \Phi(\zeta, k)|}, \\
& \quad k \in \bar{U}_{j}, \quad|k| \leq K, \quad \zeta \in \mathcal{I}, \quad t>0, \quad j=1, \cdots, 4,
\end{aligned}
$$

where the constant $C$ is independent of $\zeta, t, k$.

(2) $r_{1, a}$ and $r_{2, a}$ satisfy

$$
\left|r_{j, a}(x, t, k)\right| \leq \frac{C}{1+|k|} e^{\frac{t}{4}|\operatorname{Re} \Phi(\zeta, k)|}, \quad k \in \bar{U}_{j}, \quad \zeta \in \mathcal{I}, \quad t>0, \quad j=1,2
$$

where the constant $C$ is independent of $\zeta, t, k$.

(3) $r_{1, r}$ and $r_{2, r}$ satisfy

$$
\begin{aligned}
\left|r_{j, r}(x, t, k)\right| \leq C \frac{\left|k-k_{0}\right|}{1+|k|^{2}} t^{-3 / 2}, & k \in\left(-\infty,-k_{0}\right) \cup\left(k_{0}, \infty\right), \\
& \zeta \in \mathcal{I}, \quad t>0, \quad j=1,2,
\end{aligned}
$$

where the constant $C$ is independent of $\zeta, t, k$.

(4) $r_{3, r}$ and $r_{4, r}$ satisfy

$$
\begin{aligned}
\left|r_{j, r}(x, t, k)\right| \leq C\left|k^{2}-k_{0}^{2}\right| t^{-3 / 2}, \quad & k \in\left(-k_{0}, k_{0}\right), \\
& \zeta \in \mathcal{I}, \quad t>0, \quad j=1,2,
\end{aligned}
$$

where the constant $C$ is independent of $\zeta, t, k$.

(5) The following symmetries are valid:

$$
r_{j, a}(\zeta, t, k)=\overline{r_{j, a}(\zeta, t,-\bar{k})}, r_{j, r}(\zeta, t, k)=\overline{r_{j, r}(\zeta, t,-\bar{k})}, \quad j=1, \cdots, 4
$$

As a result, the function $m(x, t, k)$ satisfies the $\mathrm{RH}$ problem

$$
\left\{\begin{array}{l}
m_{+}(x, t, k)=m_{-}(x, t, k) v(x, t, k), \quad k \in \Gamma, \\
m(x, t, k) \rightarrow I, \quad k \rightarrow \infty
\end{array}\right.
$$




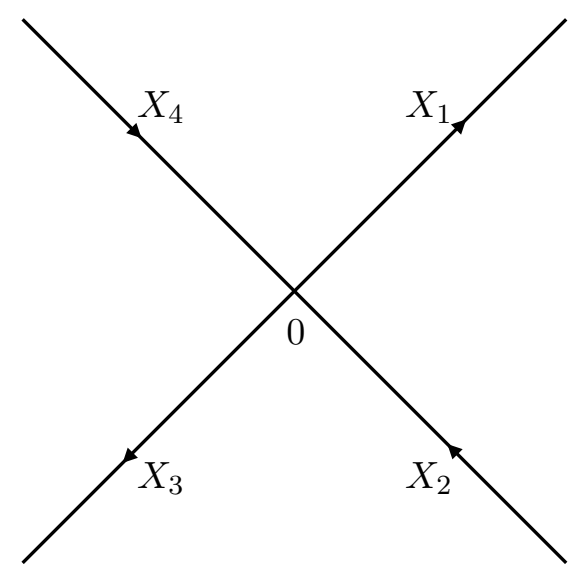

Figure 3. Contour $X$ in the complex z-plane

where the jump matrix is

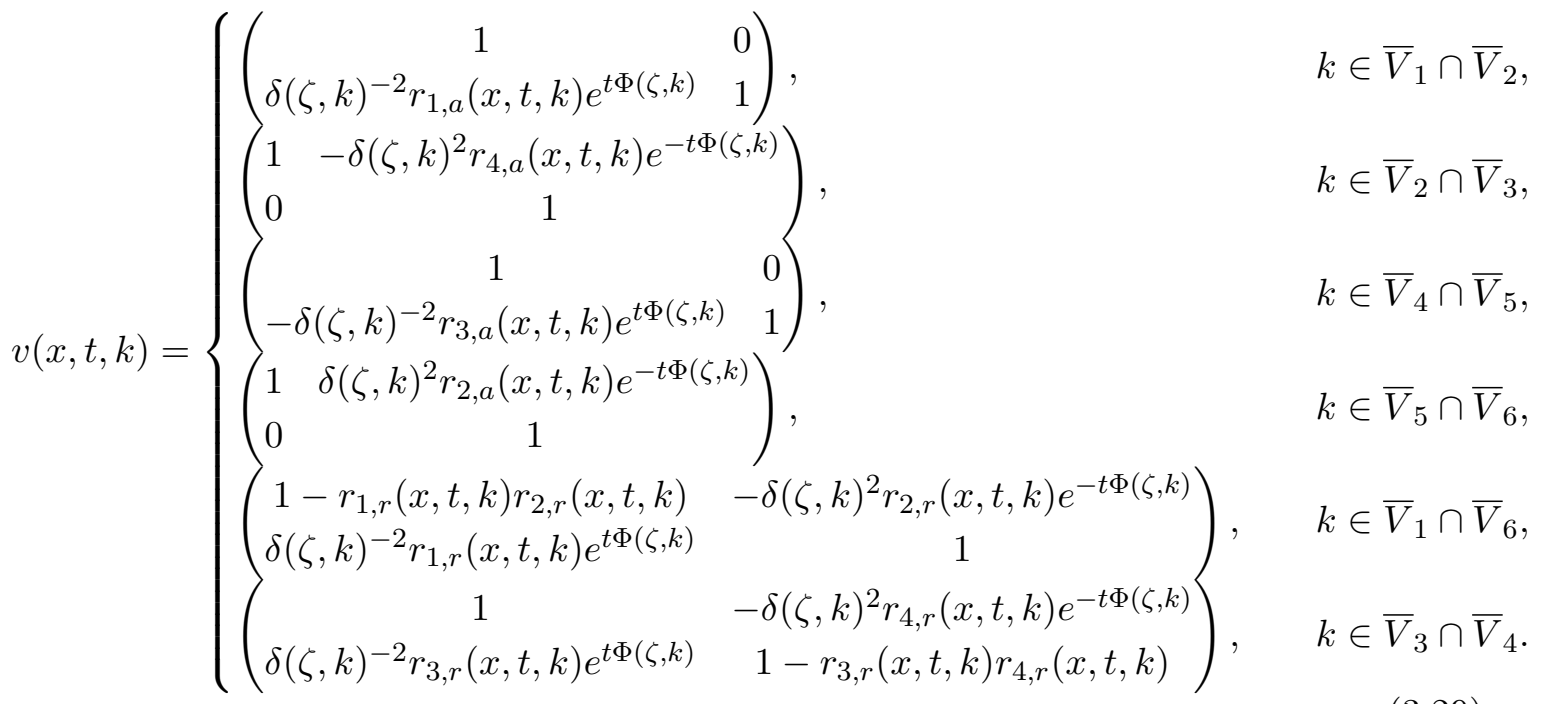

By (3.5) and (3.27), $v(\zeta, t, k)$ satisfies the symmetry

$$
v(\zeta, t, k)=\overline{v(\zeta, t,-\bar{k})}, \quad \zeta \in \mathcal{I}, \quad t>0, \quad k \in \Sigma .
$$

Step 3 Let $m^{X}(\zeta, z)$ be the solution of the $\mathrm{RH}$ problem in the complex $z$-plane:

$$
\left\{\begin{array}{l}
m^{X}(\zeta, z)_{+}=m^{X}(\zeta, z)_{-} v^{X}(\zeta, z), \quad z \in X, \\
m^{X}(\zeta, z) \rightarrow I, \quad z \rightarrow \infty
\end{array}\right.
$$

where contour $X=X_{1} \cup \cdots \cup X_{4}$ is shown in Figure 3 . 
The jump matrix is

$$
v^{X}(\zeta, z)= \begin{cases}\left(\begin{array}{cc}
1 & 0 \\
q_{1}(\zeta) z^{-2 i \nu(\zeta)} e^{\frac{i z^{2}}{2}} & 1
\end{array}\right), & z \in X_{1}, \\
\left(\begin{array}{cc}
1 & q_{2}(\zeta) z^{2 i \nu(\zeta)} e^{-\frac{i z^{2}}{2}} \\
0 & 1
\end{array}\right), & z \in X_{2}, \\
\left(\begin{array}{cc}
-\frac{q_{1}(\zeta)}{q_{1}(\zeta) q_{2}(\zeta)} z^{-2 i \nu(\zeta)} e^{\frac{i z^{2}}{2}} & 1
\end{array}\right), & z \in X_{3}, \\
\left(\begin{array}{cc}
1-\frac{q_{2}(\zeta)}{q_{1}(\zeta) q_{2}(\zeta)} z^{2 i \nu(\zeta)} e^{-\frac{i z^{2}}{2}} \\
0
\end{array}\right. & z \in X_{4} .\end{cases}
$$

We point out that in the local case (see [22]), $q_{1}$ and $q_{2}$ are defined as

$$
\begin{aligned}
q_{1}(\zeta) & =\left.\delta(\zeta, k)^{-2} r(k)\left(\frac{k-k_{0}}{\sqrt{48 k_{0}}}\right)^{2 i \nu(\zeta)}\right|_{k=k_{0}} \\
& =e^{-\chi\left(\zeta, k_{0}\right)} r\left(k_{0}\right) e^{2 i \nu(\zeta) \ln \left(2 \sqrt{48} k_{0}^{3 / 2}\right)}, \\
q_{2}(\zeta) & =\overline{q_{1}(\zeta)}
\end{aligned}
$$

By contrast, to keep the blondness of the function $q_{j}(\zeta), \zeta \in \mathcal{I}, j=1,2$ for the nonlocal case, we let

$$
\begin{aligned}
q_{1}(\zeta) & =\left.\delta(\zeta, k)^{-2} r_{1}(k)\left(\frac{2\left(k-k_{0}\right)}{k_{0}}\right)^{2 i \nu(\zeta)}\right|_{k=k_{0}} \\
& =e^{-\chi\left(\zeta, k_{0}\right)} r_{1}\left(k_{0}\right) e^{2 i \nu(\zeta) \ln 4}, \\
q_{2}(\zeta) & =\left.\delta(\zeta, k)^{2} r_{2}(k)\left(\frac{2\left(k-k_{0}\right)}{k_{0}}\right)^{-2 i \nu(\zeta)}\right|_{k=k_{0}} \\
& =e^{\chi\left(\zeta, k_{0}\right)} r_{2}\left(k_{0}\right) e^{-2 i \nu(\zeta) \ln 4} .
\end{aligned}
$$

From Lemma A.1, the unique solution $m^{X}(\zeta, z)$ of the RH problem 3.31 can be explicitly expressed in terms of parabolic-cylinder function. Together with $D(\zeta, t)$ defined by

$$
D(\zeta, t)=e^{-\frac{t \phi(\zeta, 0)}{2} \sigma_{3}} \tau^{-\frac{i \nu(\zeta)}{2} \sigma_{3}},
$$

where

$$
\begin{array}{r}
\epsilon=\frac{k_{0}}{2}, \quad \rho=\epsilon \sqrt{48 k_{0}}, \quad \tau=t \rho^{2}=12 k_{0}^{3} t, \\
\phi(\zeta, z)=\Phi\left(\zeta, k_{0}+\frac{\epsilon}{\rho} z\right)=-16 i k_{0}^{3}+\frac{i z^{2}}{2}+\frac{i z^{3}}{12 \rho},
\end{array}
$$

we use $m^{X}(\zeta, z)$ to introduce $m_{0}(\zeta, t, k)$ for $k$ near $k_{0}$ :

$$
m_{0}(\zeta, t, k)=D(\zeta, t) m^{X}\left(\zeta, \frac{\sqrt{\tau}}{\epsilon}\left(k-k_{0}\right)\right) D(\zeta, t)^{-1}, \quad\left|k-k_{0}\right| \leq \epsilon,
$$

and extend it to a neighborhood of $-k_{0}$ by symmetry:

$$
m_{0}(\zeta, t, k)=\overline{m_{0}(\zeta, t,-\bar{k})}, \quad\left|k+k_{0}\right| \leq \epsilon .
$$




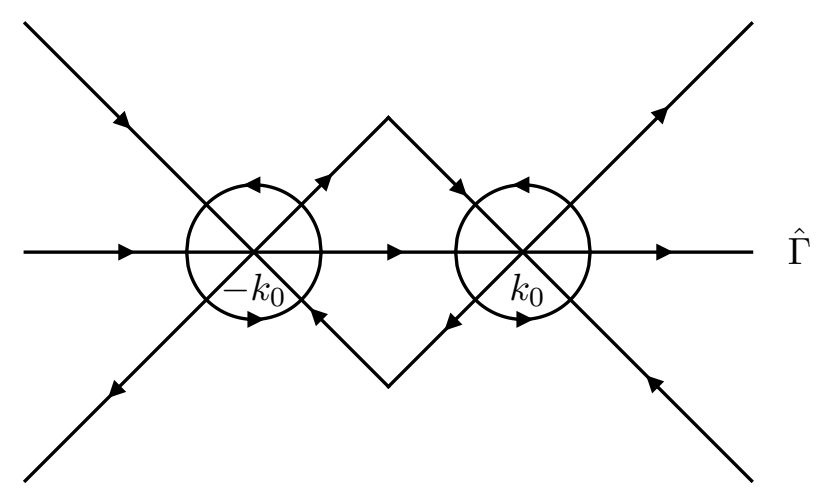

Figure 4. The jump contour $\hat{\Gamma}$

Remark 3.3. The method introduced in [22] cannot be imitated indiscriminately to deal with the situation where $\operatorname{Im} \nu(\zeta) \neq 0$. In [22] $D(\zeta, t)$ is defined by

$$
D(\zeta, t)=e^{-\frac{t \phi(\zeta, 0)}{2} \sigma_{3}} t^{-\frac{i \nu(\zeta)}{2} \sigma_{3}} .
$$

Notice (3.35), we replace $t$ by $\tau$ in (3.39) to define $D(\zeta, t)$. Actually, our adjustment including that to the function $q_{j}(\zeta), j=1,2$ (see (3.33) and (3.34)) is also valid for the study of the local case.

Then we use $m_{0}(\zeta, t, k)$ to introduce function $\hat{m}(\zeta, t, k)$ :

$$
\hat{m}(\zeta, t, k)= \begin{cases}m(\zeta, t, k) m_{0}(\zeta, t, k)^{-1}, & \left|k \pm k_{0}\right|<\epsilon, \\ m(\zeta, t, k), & \text { elsewhere. }\end{cases}
$$

By the RH problems (3.20) and the definition of $m_{0}, \hat{m}(\zeta, t, k)$ satisfies the following $\mathrm{RH}$ problem

$$
\left\{\begin{array}{l}
\hat{m}(\zeta, t, k)_{+}=\hat{m}(\zeta, t, k)_{-} \hat{v}(\zeta, t, k), \quad k \in \hat{\Gamma}, \\
\hat{m}(\zeta, t, k) \rightarrow I, \quad k \rightarrow \infty
\end{array}\right.
$$

where $\hat{\Gamma}=\Gamma \cup\left\{k|| k \pm k_{0} \mid=\epsilon\right\}$ is oriented as in Figure 4, and the jump matrix is

$$
\hat{v}(\zeta, t, k)= \begin{cases}m_{0-}(\zeta, t, k) v(\zeta, t, k) m_{0+}(\zeta, t, k)^{-1}, & \left|k_{0} \pm k_{0}\right|<\epsilon, \\ m_{0}(\zeta, t, k), & \left|k_{0} \pm k_{0}\right|=\epsilon \\ v(\zeta, t, k), & \text { elsewhere }\end{cases}
$$

The model RH problem (3.41) is finally obtained. 


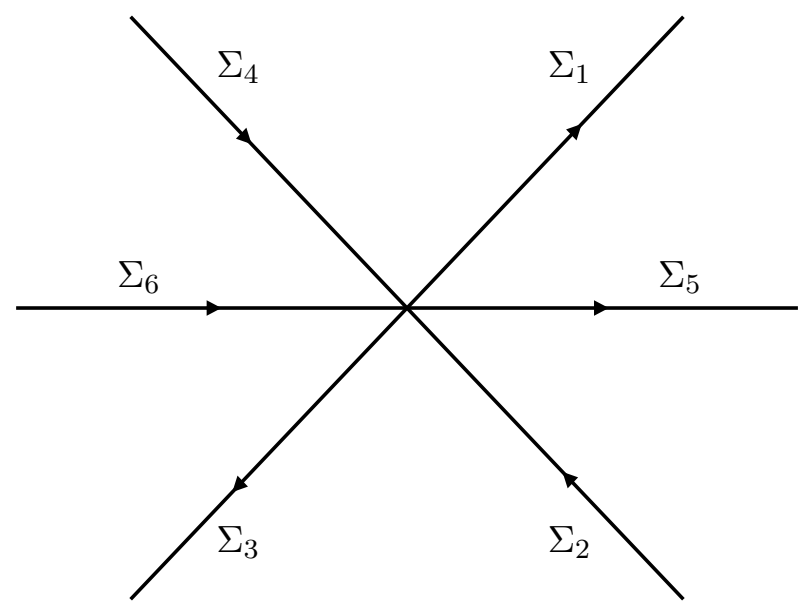

Figure 5. The contour $\Sigma=\Sigma_{1} \cup \cdots \cup \Sigma_{6}$.

\section{LONG time ASYMPtotics}

We use the model RH problem (3.41) to derive the asymptotics of the nonlocal mKdV equation in the similarity sector.

Let $\Sigma$ denote the counter $\Sigma=\Sigma_{1} \cup \cdots \cup \Sigma_{6} \subset \mathbb{C}$, where

$$
\begin{array}{ll}
\Sigma_{1}=\left\{s e^{\frac{i \pi}{4}} \mid 0 \leq s<\infty\right\}, & \Sigma_{2}=\left\{s e^{-\frac{i \pi}{4}} \mid 0 \leq s<\infty\right\}, \\
\Sigma_{3}=\left\{s e^{-\frac{3 i \pi}{4}} \mid 0 \leq s<\infty\right\}, & \Sigma_{4}=\left\{s e^{\frac{3 i \pi}{4}} \mid 0 \leq s<\infty\right\}, \\
\Sigma_{5}=\{s \mid 0 \leq s<\infty\}, & \Sigma_{6}=\{-s \mid 0 \leq s<\infty\},
\end{array}
$$

are oriented as in Figure 5. For $r>0$, we denote $\Sigma^{r}=\Sigma_{1}^{r} \cup \cdots \cup \Sigma_{6}^{r}$, where $\Sigma_{j}^{r}=$ $\Sigma_{j} \cap D(0, r), j=1, \cdots, 6$ and $D(k, r)$ is the disk of radius $r$ centered at $k$.

Lemma 4.1. Let $\Gamma_{\Sigma}= \pm k_{0}+\Sigma^{\epsilon}$ and let $\Gamma^{\prime}=\Gamma \backslash \Gamma_{\Sigma}$ as shown in Figure 6, Let $\hat{w}(\zeta, t, k)=$ $\hat{v}(\zeta, t, k)-I$, then $\hat{w}(\zeta, t, k)$ satisfies

$$
\left\{\begin{array}{l}
\|\hat{w}(\zeta, t, \cdot)\|_{L^{p}\left(\Gamma^{\prime}\right)}=O\left(\epsilon^{\frac{1}{p}} \tau^{-1}\right), \quad p=1,2, \\
\|\hat{w}(\zeta, t, \cdot)\|_{L^{\infty}\left(\Gamma^{\prime}\right)}=O\left(\tau^{-1}\right),
\end{array}\right.
$$

uniformly with respect to $\zeta \in \mathcal{I}$, as $\tau \rightarrow \infty$.

Proof. Let $\gamma$ denote the intersection of $\Gamma^{\prime}$ and the line $k_{0}+\mathbb{R} e^{\frac{i \pi}{4}}$, i.e.

$$
\gamma=\left\{k_{0}+u e^{\frac{i \pi}{4}} \mid u \in\left(-\sqrt{2} k_{0},-\frac{k_{0}}{2}\right] \cup\left[\frac{k_{0}}{2}, \infty\right)\right\}
$$



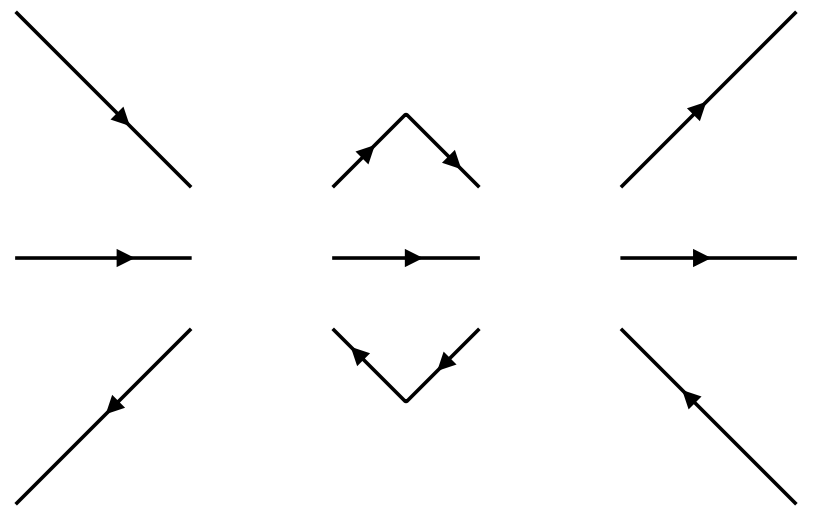

Figure 6. The contour $\Gamma^{\prime}=\Gamma \backslash \Gamma_{\Sigma^{\epsilon}}$.

Let $k=k_{0}+u e^{\frac{i \pi}{4}}$. By 3.42$)$ and $(3.29), \hat{w}$ has the following form on $\gamma$ :

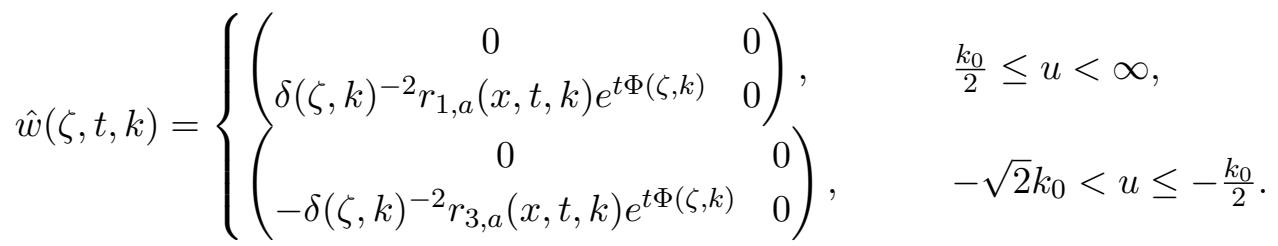

It's enough to prove that $\delta(\zeta, k)^{ \pm 1}$ is uniformly bounded on $\Gamma^{\prime}$ with respect to $\zeta \in \mathcal{I}$, i.e.

$$
\sup _{\zeta \in \mathcal{I}} \sup _{k \in \Gamma^{\prime}}\left|\delta(\zeta, k)^{ \pm 1}\right| \leq C
$$

From Lemma $3.1 \chi(\zeta, k)$ is uniformly bounded on $\gamma$ with respect to $\zeta \in \mathcal{I}$. Thus

$$
\begin{aligned}
\left|\delta(\zeta, k)^{ \pm 1}\right| & =\left|\left(\frac{k-k_{0}}{k+k_{0}}\right)^{ \pm i \nu} e^{ \pm \chi(\zeta, k)}\right| \leq C\left|\left(\frac{u e^{i \pi / 4}}{u e^{i \pi / 4}+2 k_{0}}\right)^{ \pm i \nu}\right| \\
& \leq C\left|\left(1+\frac{2 k_{0}}{u} e^{-i \pi / 4}\right)^{\mp i \nu}\right|,
\end{aligned}
$$

where $1+\frac{2 k_{0}}{u} e^{-i \pi / 4}$ satisfies the following inequalities

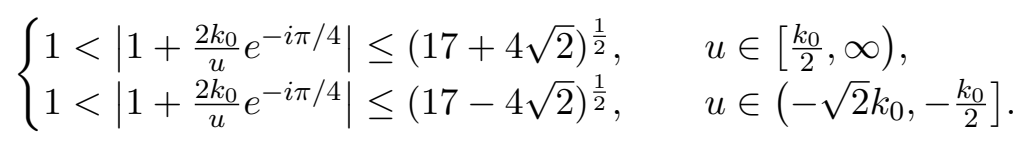

By (4.6) and (4.7), $\delta(\zeta, k)^{ \pm 1}$ is uniformly bounded on $\gamma$ with respect to $\zeta \in \mathcal{I}$. Since similar arguments apply to the remaining parts of $\Gamma^{\prime}$, this prove 4.5. 
Since the decompositions of $r_{j}\left(r_{j}=r_{j, a}+r_{j, r}, j=1, \cdots, 4\right)$ is similar to the local case, we can follow [22] to accomplish the rest of the proof by (3.23), (3.24), (3.25) and (3.26).

We normalize the jump matrix $v(\zeta, t, k)$ on $\Gamma_{\Sigma}$

$$
v_{0}(\zeta, t, z)=v\left(\zeta, t, k_{0}+\frac{\epsilon z}{\rho}\right), \quad z \in \Sigma^{\rho}
$$

which has the form of

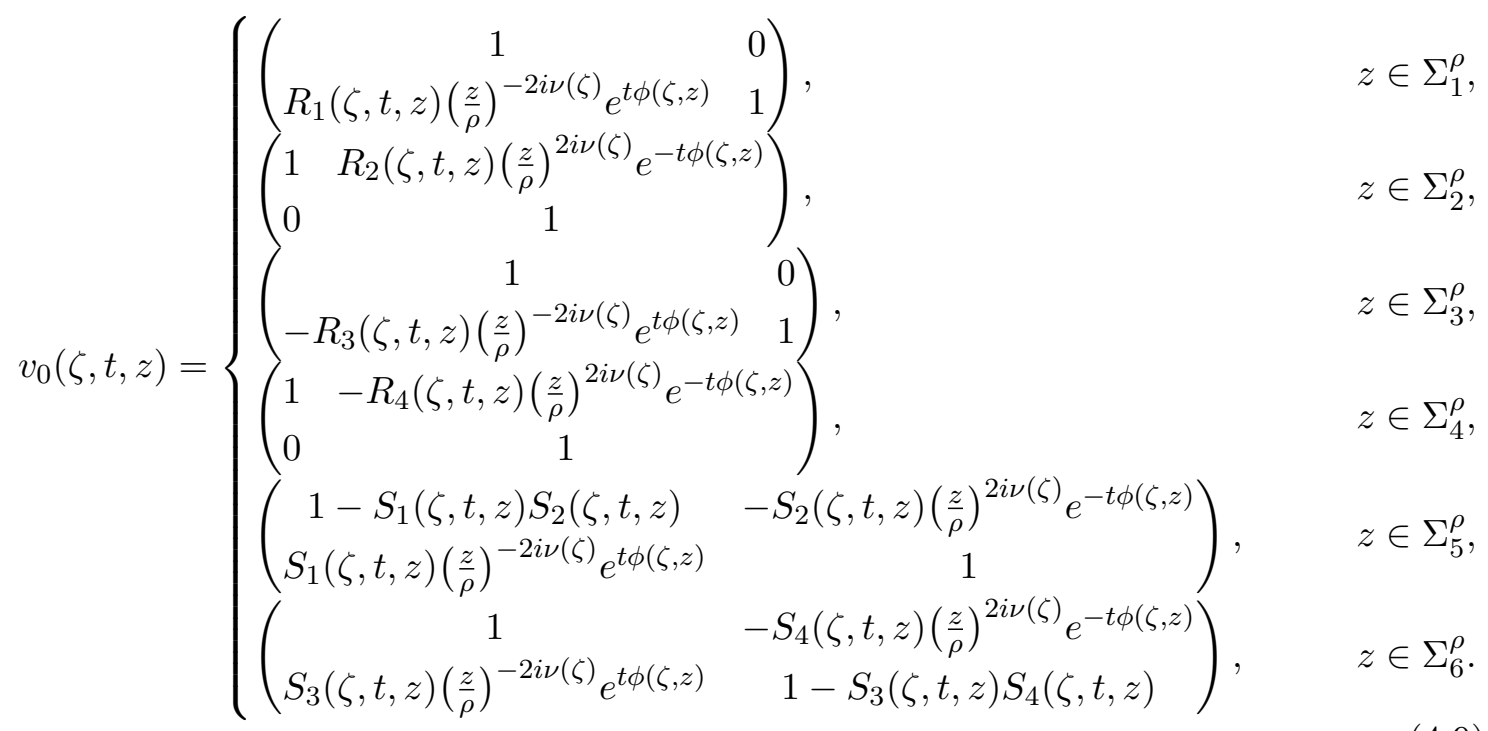

where $\epsilon, \rho$ and $\phi$ are defined by (3.36). The phase $\phi(\zeta, z)$ is identical to the local case, which is a smooth function of $(\zeta, z) \in \mathcal{I} \times \mathbb{C}$ satisfying condition (2.10) and (2.11) of Theorem 2.1 in [22]. Moreover, $\left\{R_{j}(\zeta, t, k)\right\}_{1}^{4}$ and $\left\{S_{j}(\zeta, t, k)\right\}_{1}^{4}$ satisfy the following Lemma.

Lemma 4.2. There exist constants $(\alpha, L) \in[1 / 2,1) \times(0, \infty)$ such that the functions $\left\{R_{j}(\zeta, t, z)\right\}_{1}^{4}$ and $\left\{S_{j}(\zeta, t, k)\right\}_{1}^{4}$ satisfy the inequalities:

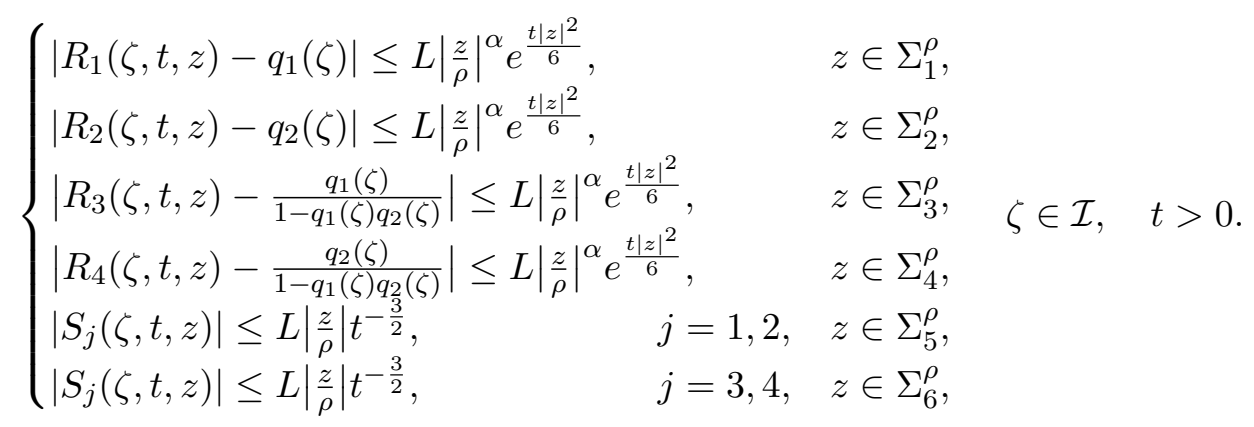

where $q_{1}(\zeta)$ and $q_{2}(\zeta)$ are defined by 3.34.). 
Proof. Equations (3.29) and (4.9) imply that

$$
\begin{cases}R_{1}(\zeta, t, z)=\delta(\zeta, k)^{-2} r_{1, a}(x, t, k)\left(\frac{z}{\rho}\right)^{2 i \nu(\zeta)}, & R_{2}(\zeta, t, z)=\delta(\zeta, k)^{2} r_{2, a}(x, t, k)\left(\frac{z}{\rho}\right)^{-2 i \nu(\zeta)} \\ R_{3}(\zeta, t, z)=\delta(\zeta, k)^{-2} r_{3, a}(x, t, k)\left(\frac{z}{\rho}\right)^{2 i \nu(\zeta)}, & R_{4}(\zeta, t, z)=\delta(\zeta, k)^{2} r_{4, a}(x, t, k)\left(\frac{z}{\rho}\right)^{-2 i \nu(\zeta)} \\ S_{1}(\zeta, t, z)=\delta(\zeta, k)^{-2} r_{1, r}(x, t, k)\left(\frac{z}{\rho}\right)^{2 i \nu(\zeta)}, & S_{2}(\zeta, t, z)=\delta(\zeta, k)^{2} r_{2, r}(x, t, k)\left(\frac{z}{\rho}\right)^{-2 i \nu(\zeta)} \\ S_{3}(\zeta, t, z)=\delta(\zeta, k)^{-2} r_{3, r}(x, t, k)\left(\frac{z}{\rho}\right)^{2 i \nu(\zeta)}, & S_{4}(\zeta, t, z)=\delta(\zeta, k)^{2} r_{4, r}(x, t, k)\left(\frac{z}{\rho}\right)^{-2 i \nu(\zeta)}\end{cases}
$$

Let $k=k_{0}+\frac{\epsilon}{\rho} z$. Using the expression 3.11 , we can write

$$
R_{1}(\zeta, t, z)=e^{-2 \chi(\zeta, k)} r_{1, a}(x, t, k) e^{2 i \nu(\zeta) \ln \left(\frac{2\left(k+k_{0}\right)}{k_{0}}\right)}, \quad z \in \Sigma_{1}^{\rho} .
$$

By 3.23 , we find

$$
\begin{aligned}
R_{1}(\zeta, t, 0) & =e^{-2 \chi\left(\zeta, k_{0}\right)} r_{1}\left(k_{0}\right) e^{2 i \nu(\zeta) \ln 4} \\
& =q_{1}(\zeta)
\end{aligned}
$$

Similar arguments imply that

$$
R_{2}(\zeta, t, 0)=q_{2}(\zeta), \quad R_{3}(\zeta, t, 0)=\frac{q_{1}(\zeta)}{1-q_{1}(\zeta) q_{2}(\zeta)}, \quad R_{4}(\zeta, t, 0)=\frac{q_{2}(\zeta)}{1-q_{1}(\zeta) q_{2}(\zeta)} .
$$

Note that $z \in \Sigma_{1}^{\rho}$ is equivalent to $k \in k_{0}+\Sigma_{1}^{\epsilon}$. For $z \in \Sigma_{1}^{\rho}$, we have

$$
\begin{aligned}
\left|R_{1}(\zeta, t, z)-q_{1}(\zeta)\right| & \leq\left|e^{-2 \chi(\zeta, k)}-e^{-2 \chi\left(\zeta, k_{0}\right)}\right|\left|r_{1, a}(x, t, k) e^{2 i \nu(\zeta) \ln \left(\frac{2\left(k+k_{0}\right)}{k_{0}}\right)}\right| \\
& +\left|e^{-2 \chi\left(\zeta, k_{0}\right)}\right|\left|r_{1, a}(x, t, k)-r_{1}\left(k_{0}\right)\right|\left|e^{2 i \nu(\zeta) \ln \left(\frac{2\left(k+k_{0}\right)}{k_{0}}\right)}\right| \\
& +\left|e^{-2 \chi\left(\zeta, k_{0}\right)} r_{1}\left(k_{0}\right)\right|\left|1-e^{-2 i \nu(\zeta) \ln \left(\frac{k+k_{0}}{2 k_{0}}\right)}\right|\left|e^{2 i \nu(\zeta) \ln \left(\frac{2\left(k+k_{0}\right)}{k_{0}}\right)}\right| .
\end{aligned}
$$

From Lemma 3.1, $e^{2 \chi\left(\zeta, k_{0}\right)}$ is bounded for $\zeta \in \mathcal{I}$. Moreover, let $k=k_{0}+u e^{i \pi / 4}$, we have

$$
\left|e^{2 i \nu(\zeta) \ln \left(\frac{2\left(k+k_{0}\right)}{k_{0}}\right)}\right|=\left|\left(4+\frac{2 u}{k_{0}} e^{i \pi / 4}\right)^{2 i \nu(\zeta)}\right|,
$$

where $4+\frac{2 u}{k_{0}} e^{i \pi / 4}$ satisfies

$$
(17-4 \sqrt{2})^{\frac{1}{2}}<\left|4+\frac{2 u}{k_{0}} e^{i \pi / 4}\right| \leq 4, \quad 0 \leq u<\epsilon .
$$

Thus $e^{2 i \ln \left(\frac{2\left(k+k_{0}\right)}{k_{0}}\right)}$ is uniformly bounded with respect to $\zeta \in \mathcal{I}$ and $k \in k_{0}+\Sigma_{1}^{\epsilon}$.

As in the local case, using (3.23), (3.24) and the estimate

$$
\left|\operatorname{Re} \phi\left(\zeta, v e^{i \pi / 4}\right)\right| \leq \frac{2 v^{2}}{3}, \quad 0 \leq v<\rho,
$$

we can prove that for $z \in \Sigma_{1}^{\rho}$, the following inequalities holds:

$$
\begin{aligned}
& \left|r_{1, a}(x, t, k)-r_{1}\left(k_{0}\right)\right| \leq C \frac{\epsilon|z|}{\rho} e^{\frac{t}{6}|z|^{2}} \\
& \left|r_{1, a}(x, t, k)\right| \leq C e^{\frac{t}{6}|z|^{2}}
\end{aligned}
$$


Thus,

$$
\begin{aligned}
\left|R_{1}(\zeta, t, z)-q_{1}(\zeta)\right| & \leq C e^{\frac{t|z|^{2}}{6}}\left|e^{-2 \chi(\zeta, k)}-e^{-2 \chi\left(\zeta, k_{0}\right)}\right|+C \frac{\epsilon|z|}{\rho} e^{\frac{t|z|^{2}}{6}} \\
& +C\left|1-e^{-2 i \nu(\zeta) \ln \left(\frac{k+k_{0}}{2 k_{0}}\right)}\right|
\end{aligned}
$$

Employing (3.12), we have

$$
\left|\chi(\zeta, k)-\chi\left(\zeta, k_{0}\right)\right| \leq C\left|\ln \left(\frac{k_{0}+k}{2 k_{0}}\right)\right|+C\left|\int_{-k_{0}}^{k_{0}} \ln \left(\frac{s-k}{s-k_{0}}\right) d \ln \left(1-r_{1}(s) r_{2}(s)\right)\right| .
$$

Then we can follow the proof of Lemma 4.9 in [22] to derive the following inequalities

$$
\begin{aligned}
& \left|1-e^{-2 i \nu(\zeta) \ln \left(\frac{k+k_{0}}{2 k_{0}}\right)}\right| \leq C k_{0}^{-1}\left|k-k_{0}\right|, \\
& \left|e^{-2 \chi(\zeta, k)}-e^{-2 \chi\left(\zeta, k_{0}\right)}\right| \leq C\left|k-k_{0}\right|\left(k_{0}^{-1}+|\ln | k-k_{0}||\right) .
\end{aligned}
$$

Notice that 4.19 is slightly different from Lemma 4.9 in [22], which is caused by the first term of right-hand side of 4.17).

Using 4.16), 4.18 and 4.19, We can verify 4.10 in the case of $z \in \Sigma_{1}^{\rho}$; the case of $z \in \Sigma_{j}^{\rho}, j=2,3,4$ are similar. Using (3.25), (3.26) and the uniform boundness of $e^{-2 \chi(\zeta, k)}$ and $e^{2 i \ln \left(\frac{2\left(k+k_{0}\right)}{k_{0}}\right)}$, it's clear that $S_{j}(\zeta, t, z), j=1, \cdots, 4$ satisfy the inequalities in 4.10.

Lemma 4.3. On $\Gamma_{\Sigma}$, function $\hat{w}$ satisfies

$$
\begin{aligned}
\hat{w}(\zeta, t, k)=O\left(\tau^{-\frac{\alpha}{2}+|\operatorname{Im} \nu(\zeta)|} e^{-\frac{\tau}{24 \epsilon^{2}}\left|k \mp k_{0}\right|^{2}}\right), & \\
\tau & \rightarrow \infty, \quad \zeta \in \mathcal{I}, \quad k \in \pm k_{0}+\bigcup_{j=1}^{4} \Sigma_{j}^{\epsilon},
\end{aligned}
$$

and

$$
\hat{w}(\zeta, t, k)=O\left(\tau^{-\frac{3}{2}+2|\operatorname{Im} \nu(\zeta)|}\right), \quad \tau \rightarrow \infty, \quad \zeta \in \mathcal{I}, \quad k \in \pm k_{0}+\bigcup_{j=5}^{6} \Sigma_{j}^{\epsilon},
$$

where the error term is uniform with respect to $\zeta \in \mathcal{I}$ and $k \in \Gamma_{\Sigma}$.

Proof. We prove the case of $k \in k_{0}+\Sigma^{\epsilon}$. Symmetries (3.30) and $(3.38)$ implies that

$$
\hat{w}(\zeta, t, k)=\overline{\hat{w}(\zeta, t,-\bar{k})}, \quad \zeta \in \mathcal{I}, \quad t>0, \quad k \in \hat{\Gamma} .
$$

Thus the case of $k \in-k_{0}+\Sigma^{\epsilon}$ follows by the above symmetry. We let $k=k_{0}+\frac{\epsilon}{\rho} z, z \in \Sigma^{\rho}$. Then

$$
\begin{aligned}
\hat{w}(\zeta, t, k) & =m_{0-}(\zeta, t, k) v(\zeta, t, k) m_{0+}(\zeta, t, k)^{-1}-I \\
& =D(\zeta, t) m_{-}^{X}(\zeta, \sqrt{t} z) D(\zeta, t)^{-1} v_{0}(\zeta, t, z) D(\zeta, t) m_{+}^{X}(\zeta, \sqrt{t} z)^{-1} D(\zeta, t)^{-1}-I
\end{aligned}
$$


By Lemma A.1, there exists a constant $G$ such that $m_{ \pm}^{X}(\zeta, \sqrt{t} z)$ is uniformly bounded with respect to $\zeta \in \mathcal{I}$ and $|\sqrt{t} z| \geq G ; m_{ \pm}^{X}(\zeta, \sqrt{t} z)(\sqrt{t} z)^{i \nu(\zeta) \sigma_{3}}$ is uniformly bounded with respect to $\zeta \in \mathcal{I}$ and $|\sqrt{t} z|<G$. Thus we write $\hat{w}$ as $\hat{w}(\zeta, t, k)= \begin{cases}D(\zeta, t) m_{-}^{X}(\zeta, \sqrt{t} z) u_{1}(\zeta, t, z) m_{+}^{X}(\zeta, \sqrt{t} z)^{-1} D(\zeta, t)^{-1}, & |\sqrt{t} z| \geq G, \\ D(\zeta, t) m_{-}^{X}(\zeta, \sqrt{t} z)\left((\sqrt{t} z)^{i \nu(\zeta) \hat{\sigma}_{3}} u_{2}(\zeta, t, z)\right) m_{+}^{X}(\zeta, \sqrt{t} z)^{-1} D(\zeta, t)^{-1}, & |\sqrt{t} z|<G .\end{cases}$

where

$$
\begin{aligned}
& u_{1}(\zeta, t, z)=D(\zeta, t)^{-1} v_{0}(\zeta, t, z) D(\zeta, t)-v^{X}(\zeta, \sqrt{t} z) \\
& u_{2}(\zeta, t, z)=(\sqrt{t} z)^{-i \nu(\zeta) \hat{\sigma}_{3}} u_{1}(\zeta, t, z)
\end{aligned}
$$

By 3.35$),|D(\zeta, t)|=O\left(\tau^{\frac{|\operatorname{Im} \nu(\zeta)|}{2}}\right)$. Consequently, it's enough to prove that

$$
\begin{aligned}
u_{1}(\zeta, t, z)=O\left(\tau^{-\frac{\alpha}{2}} e^{-\frac{t|z|^{2}}{24}}\right), \quad \tau \rightarrow \infty, \quad \zeta \in \mathcal{I}, \quad z \in \bigcup_{j=1}^{4} \Sigma_{j}^{\rho}, \quad|\sqrt{t} z| \geq G, \\
u_{1}(\zeta, t, z)=O\left(\tau^{-\frac{3}{2}+|\operatorname{Im} \nu(\zeta)|}\right), \quad \tau \rightarrow \infty, \quad \zeta \in \mathcal{I}, \quad z \in \bigcup_{j=5}^{6} \Sigma_{j}^{\rho}, \quad|\sqrt{t} z| \geq G, \quad \tau^{4}, \quad \zeta \in \mathcal{I}, \quad z \in \bigcup_{j=1}^{4} \Sigma_{j}^{\rho}, \quad|\sqrt{t} z|<G, \\
u_{2}(\zeta, t, z)=O\left(\tau^{-\frac{\alpha}{2}} e^{-\frac{t|z|^{2}}{24}}\right), \quad \tau \rightarrow \infty, \quad \zeta \in \mathcal{I}, \quad z \in \bigcup_{j=5}^{6} \Sigma_{j}^{\rho}, \quad|\sqrt{t} z|<G
\end{aligned}
$$

uniformly with respect to $(\zeta, z)$ in the given ranges.

For the case of $z \in \Sigma_{1}^{\rho}$, we have

$$
u_{1}(\zeta, t, z)=\left(\begin{array}{cc}
0 & 0 \\
\left(R_{1}(\zeta, t, z) e^{t(\phi(\zeta, z)-\phi(\zeta, 0))}-q_{1}(\zeta) e^{\frac{i t z^{2}}{2}}\right)(\sqrt{t} z)^{-2 i \nu(\zeta)} & 0
\end{array}\right) .
$$

So only the (21) entry of $u_{1}(\zeta, t, z)$ is nonzero, and for $|\sqrt{t} z| \geq G$ we find that

$$
\begin{aligned}
\left|\left(u_{1}(\zeta, t, z)\right)_{21}\right| & =\left|R_{1}(\zeta, t, z) e^{t(\phi(\zeta, z)-\phi(\zeta, 0))}-q_{1}(\zeta) e^{\frac{i t z^{2}}{2}}\right|\left|(\sqrt{t} z)^{-2 i \nu(\zeta)}\right| \\
& =\left|R_{1}(\zeta, t, z) e^{t \hat{\phi}(\zeta, z)}-q_{1}(\zeta)\right| e^{-\frac{t|z|^{2}}{2}}|\sqrt{t} z|^{2 \operatorname{Im} \nu(\zeta)} e^{\frac{\pi}{2} \operatorname{Re} \nu(\zeta)} \\
& \leq C\left(\left|R_{1}(\zeta, t, z)-q_{1}(\zeta)\right| e^{t \operatorname{Re} \hat{\phi}(\zeta, z)}+|q(\zeta)|\left|e^{t \hat{\phi}(\zeta, z)}-1\right|\right) e^{-\frac{t|z|^{2}}{2}}|\sqrt{t} z|^{2 \operatorname{Im} \nu(\zeta)}
\end{aligned}
$$

where $\hat{\phi}(\zeta, z)=\phi(\zeta, z)-\phi(\zeta, 0)-\frac{i z^{2}}{2}$. As in the local case[22], we can use the inequalities

$$
\begin{aligned}
& \left|e^{w}-1\right| \leq|w| \max \left(1, e^{\operatorname{Re} w}\right), \quad w \in \mathbb{C}, \\
& \operatorname{Re} \hat{\phi}(\zeta, z) \leq \frac{|z|^{2}}{4}, \quad \zeta \in \mathcal{I}, \quad z \in \Sigma_{1}^{\rho},
\end{aligned}
$$


and the boundness of $q_{j}(\zeta), j=1,2$ to find that

$$
\begin{aligned}
&\left|\left(u_{1}(\zeta, t, z)\right)_{21}\right| \leq C\left(\left|R_{1}(\zeta, t, z)-q_{1}(\zeta)\right|+C t|\hat{\phi}(\zeta, z)|\right) e^{-\frac{t|z|^{2}}{4}}|\sqrt{t} z|^{2 \operatorname{Im} \nu(\zeta)} \\
& \zeta \in \mathcal{I}, \quad t>0, \quad z \in \Sigma_{1}^{\rho}, \quad|\sqrt{t} z| \geq G
\end{aligned}
$$

It's easy to verify that

$$
|\hat{\phi}(\zeta, z)| \leq C \frac{|z|^{3}}{\rho}, \quad \zeta \in \mathcal{I}, \quad z \in \Sigma^{\rho}
$$

so together with Lemma 4.2 , the right-hand of 4.32 is of order

$$
\begin{aligned}
& O\left(\left(\frac{L|z|^{\alpha} e^{\frac{t|z|^{2}}{6}}}{\rho^{\alpha}}+\frac{C t|z|^{3}}{\rho}\right) e^{-\frac{t|z|^{2}}{4}}\left(t|z|^{2}\right)^{\operatorname{Im} \nu(\zeta)}\right) \\
& =O\left(\left(\frac{\left(t|z|^{2}\right)^{\alpha / 2+\operatorname{Im} \nu(\zeta)}}{\tau^{\alpha / 2}}+\frac{\left(t|z|^{2}\right)^{3 / 2+\operatorname{Im} \nu(\zeta)}}{\tau^{1 / 2}}\right) e^{-\frac{t|z|^{2}}{12}}\right) \\
& =O\left(\left(\frac{1}{\tau^{\alpha / 2}}+\frac{1}{\tau^{1 / 2}}\right) e^{-\frac{t|z|^{2}}{24}}\right), \quad \tau \rightarrow \infty, \quad \zeta \in \mathcal{I}, \quad z \in \Sigma_{1}^{\rho}, \quad|\sqrt{t} z| \geq G .
\end{aligned}
$$

uniformly with respect to $(\zeta, z)$ in the given ranges. This proves 4.26 for $z \in \Sigma_{1}^{\rho}$; the cases of $z \in \Sigma_{j}^{\rho}, j=2,3,4$, are similar. For the case of $z \in \Sigma_{5}^{\rho}$, we have

$u_{1}(\zeta, t, z)=\left(\begin{array}{cc}-S_{1}(\zeta, t, z) S_{2}(\zeta, t, z) & -S_{2}(\zeta, t, z) e^{-t(\phi(\zeta, z)-\phi(\zeta, 0))}(\sqrt{t} z)^{2 i \nu(\zeta)} \\ S_{1}(\zeta, t, z) e^{t(\phi(\zeta, z)-\phi(\zeta, 0))}(\sqrt{t} z)^{-2 i \nu(\zeta)} & 0\end{array}\right.$.

By Lemma 4.2 , the (11) entry satisfies

$$
\begin{aligned}
\left|\left(u_{1}(\zeta, t, z)\right)_{11}\right|= & \left|S_{1}(\zeta, t, z) S_{2}(\zeta, t, z)\right| \leq C\left|\frac{z}{\rho}\right|^{2} t^{-3} \\
& \leq C \frac{|z|^{2}}{\tau} t^{-2} \leq C \tau^{-3}, \quad \zeta \in \mathcal{I}, \quad t>0, \quad z \in \Sigma_{5}^{\rho},
\end{aligned}
$$

and the (12) entry satisfies

$$
\begin{aligned}
\left|\left(u_{1}(\zeta, t, z)\right)_{12}\right| & =\left|S_{2}(\zeta, t, z) e^{-t(\phi(\zeta, z)-\phi(\zeta, 0))}(\sqrt{t} z)^{2 i \nu(\zeta)}\right| \\
& \leq C \frac{|z|^{1-2 \operatorname{Im} \nu(\zeta)}}{\rho} t^{-\frac{3}{2}-\operatorname{Im} \nu(\zeta)} \leq C \frac{|z|^{1-2 \operatorname{Im} \nu(\zeta)}}{\tau^{\frac{1}{2}}} t^{-1-\operatorname{Im} \nu(\zeta)}
\end{aligned}
$$

Because $-\frac{1}{2}<\operatorname{Im} \nu(\zeta)<\frac{1}{2},\left(u_{1}(\zeta, t, z)\right)_{12}$ is of order

$$
\left(u_{1}(\zeta, t, z)\right)_{12}=O\left(\tau^{-\frac{3}{2}-\operatorname{Im} \nu(\zeta)}\right), \quad \tau \rightarrow \infty, \quad \zeta \in \mathcal{I}, \quad z \in \Sigma_{5}^{\rho} .
$$

Similarly, $\left(u_{1}(\zeta, t, z)\right)_{21}$ is of order

$$
\left(u_{1}(\zeta, t, z)\right)_{21}=O\left(\tau^{-\frac{3}{2}+\operatorname{Im} \nu(\zeta)}\right), \quad \tau \rightarrow \infty, \quad \zeta \in \mathcal{I}, \quad z \in \Sigma_{5}^{\rho} .
$$

Using 4.35, 4.36 and 4.37, we prove 4.27) for $z \in \Sigma_{5}^{\rho}$; the case of $z \in \Sigma_{6}^{\rho}$ is similar.

On the other hand, (4.28) and 4.29 , the estimates of $u_{2}(\zeta, t, z)$, can be proved in the same way. 
Following Lemma 2.6 in [22, we use Lemma 4.1 and Lemma 4.3 to obtain the estimates:

$$
\begin{aligned}
\|\hat{w}(\zeta, t, \cdot)\|_{L^{2}(\hat{\Gamma})}=O\left(\epsilon^{\frac{1}{2}} \tau^{-\frac{\alpha}{2}+|\operatorname{Im} \nu(\zeta)|}\right), & \tau \rightarrow \infty, \quad \zeta \in \mathcal{I}, \\
\|\hat{w}(\zeta, t, \cdot)\|_{L^{\infty}(\hat{\Gamma})}=O\left(\tau^{-\frac{\alpha}{2}+|\operatorname{Im} \nu(\zeta)|}\right), & \tau \rightarrow \infty, \quad \zeta \in \mathcal{I}, \\
\|\hat{w}(\zeta, t, \cdot)\|_{L^{p}\left( \pm k_{0}+\Sigma^{\epsilon}\right)}=O\left(\epsilon^{\frac{1}{p}} \tau^{-\frac{1}{2 p}-\frac{\alpha}{2}+|\operatorname{Im} \nu(\zeta)|}\right), & \tau \rightarrow \infty, \quad \zeta \in \mathcal{I},
\end{aligned}
$$

where $p \in[1, \infty)$ and the error terms are uniform with respect to $\zeta \in \mathcal{I}$. Moreover, if taking acocunt of the first and second columns of $\hat{w}(\zeta, t, k)$ respectively in Lemma 4.3 , we have

$$
\begin{array}{rrr}
\left\|\hat{w}^{(j)}(\zeta, t, \cdot)\right\|_{L^{2}(\hat{\Gamma})}=O\left(\epsilon^{\frac{1}{2}} \tau^{-\frac{\alpha}{2}+(-1)^{j} \operatorname{Im} \nu(\zeta)}\right), & \tau \rightarrow \infty, \quad \zeta \in \mathcal{I}, \quad j=1,2 \\
\left\|\hat{w}^{(j)}(\zeta, t, \cdot)\right\|_{L^{\infty}(\hat{\Gamma})}=O\left(\tau^{-\frac{\alpha}{2}+(-1)^{j} \operatorname{Im} \nu(\zeta)}\right), & \tau \rightarrow \infty, \quad \zeta \in \mathcal{I}, \quad j=1,2 \\
\left\|\hat{w}^{(j)}(\zeta, t, \cdot)\right\|_{L^{p}\left( \pm k_{0}+\Sigma^{\epsilon}\right)}=O\left(\epsilon^{\frac{1}{p}} \tau^{-\frac{1}{2 p}-\frac{\alpha}{2}+(-1)^{j} \operatorname{Im} \nu(\zeta)}\right), & \tau \rightarrow \infty, \quad \zeta \in \mathcal{I}, \quad j=1,2
\end{array}
$$

Lemma 4.4. The RH problem (3.41) has a unique solution for all sufficiently large $\tau$. And for any $\alpha \in(\lambda, 1)$ this solution satisfies

$$
\begin{aligned}
& \lim _{k \rightarrow \infty}(k \hat{m}(\zeta, t, k))_{12}=-\frac{2 i \epsilon \operatorname{Re} \beta(\zeta, t)}{\tau^{\frac{1}{2}-\operatorname{Im} \nu(\zeta)}}+O\left(\epsilon \tau^{-\frac{1+\alpha}{2}+|\operatorname{Im} \nu(\zeta)|+\operatorname{Im} \nu(\zeta)}\right) \\
& \tau \rightarrow \infty, \quad \zeta \in \mathcal{I}
\end{aligned}
$$

where $\lambda=\max \left(\frac{1}{2}, \sup _{\zeta \in \mathcal{I}} 2|\operatorname{Im} \nu(\zeta)|\right)$, and the error term is uniform with respect to $\zeta \in \mathcal{I}$ and $\beta(\zeta, t)$ is defined by

$$
\beta(\zeta, t)=\frac{\sqrt{2 \pi} e^{i \pi / 4} e^{-\pi \nu(\zeta) / 2}}{q_{1}(\zeta) \Gamma(-i \nu(\zeta))} e^{-t \phi(\zeta, 0)} \tau^{-i \operatorname{Re} \nu(\zeta)} .
$$

Proof. We define the integral operator $\hat{\mathcal{C}}_{\hat{w}}: L^{2}(\hat{\Gamma})+L^{\infty}(\hat{\Gamma}) \rightarrow L^{2}(\hat{\Gamma})$ by $\hat{\mathcal{C}}_{\hat{w}} f=\hat{\mathcal{C}}_{-}(f \hat{w})$, where $\hat{\mathcal{C}}_{-}(f \hat{w})$ is the boundary value of $\hat{\mathcal{C}}(f \hat{w})$ from the right side of $\hat{\Gamma}$, and $\hat{\mathcal{C}}$ is the Cauchy operator associated with $\hat{\Gamma}$ :

$$
(\hat{\mathcal{C}} f)(z)=\frac{1}{2 \pi i} \int_{\hat{\Gamma}} \frac{f(s)}{s-z} d s, \quad z \in \mathbb{C} \backslash \hat{\Gamma} .
$$

By 4.39,

$$
\left\|\hat{\mathcal{C}}_{\hat{w}}\right\|_{\mathcal{B}\left(L^{2}(\hat{\Gamma})\right)} \leq C\|\hat{w}\|_{L^{\infty}(\hat{\Gamma})}=O\left(\tau^{-\frac{\alpha}{2}+|\operatorname{Im} \nu(\zeta)|}\right), \quad \tau \rightarrow \infty, \quad \zeta \in \mathcal{I}
$$

Since $\alpha \in(\lambda, 1),\left\|\hat{\mathcal{C}}_{\hat{w}}\right\|_{\mathcal{B}\left(L^{2}(\hat{\Gamma})\right)}$ decays to 0 as $\tau \rightarrow \infty$. Thus, there exists a $T>0$ such that $I-\hat{\mathcal{C}}_{\hat{w}(\zeta, t, \cdot)} \in \mathcal{B}\left(L^{2}(\hat{\Gamma})\right)$ is invertible for all $(\zeta, t) \in(0, \infty)$ with $\tau>T$.

Moreover, by 4.38 we have

$$
\|\hat{\mu}-I\|_{L^{2}(\hat{\Gamma})}=O\left(\epsilon^{\frac{1}{2}} \tau^{-\frac{\alpha}{2}+|\operatorname{Im} \nu(\zeta)|}\right), \quad \tau \rightarrow \infty, \quad \zeta \in \mathcal{I} .
$$


where $\hat{\mu}-I=\left(I-\hat{\mathcal{C}}_{\hat{w}}\right)^{-1} \hat{\mathcal{C}}_{\hat{w}} I \in L^{2}(\hat{\Gamma})$ is the solution of the integral equation

$$
\left(I-\hat{\mathcal{C}}_{\hat{w}}\right)(\mu-I)=\hat{\mathcal{C}}_{\hat{w}} I
$$

Consequently, by Lemma 2.9 in [22], there exists a unique solution $\hat{m}$ of the $\mathrm{RH}$ problem (3.41) whenever $\tau>T$, and in accordance with the local case, we can represent $\hat{m}$ as

$$
\hat{m}(\zeta, t, k)=I+\hat{\mathcal{C}}(\hat{\mu} \hat{w})=I+\frac{1}{2 \pi i} \int_{\hat{\Gamma}} \hat{\mu}(\zeta, t, s) \hat{w}(\zeta, t, s) \frac{d s}{s-k} .
$$

Finally, Lemma 2.10 in 22 and symmetry 4.22 imply that

$$
\begin{aligned}
& \lim _{k \rightarrow \infty} k(\hat{m}(\zeta, t, k)-I)=-\frac{1}{2 \pi i} \int_{\hat{\Gamma}} \hat{\mu}(\zeta, t, k) \hat{w}(\zeta, t, k) d k \\
& =-\frac{1}{2 \pi i}\left(\int_{\left|k-k_{0}\right|=\epsilon}+\int_{\left|k+k_{0}\right|=\epsilon}\right) \hat{\mu}(\zeta, t, k) \hat{w}(\zeta, t, k) d k-\frac{1}{2 \pi i} \int_{\Gamma} \hat{\mu}(\zeta, t, k) \hat{w}(\zeta, t, k) d k \\
& =-\frac{1}{\pi i} \operatorname{Re}\left(\int_{\left|k-k_{0}\right|=\epsilon} \hat{\mu}(\zeta, t, k)\left(m_{0}(\zeta, t, k)^{-1}-I\right) d k\right)-\frac{1}{2 \pi i} \int_{\Gamma} \hat{\mu}(\zeta, t, k) \hat{w}(\zeta, t, k) d k
\end{aligned}
$$

By Lemma A.1, we have

$$
\begin{aligned}
\left(m_{0}(\zeta, t, k)^{-1}\right)^{(2)} & =\left(D(\zeta, t) m^{X}\left(\zeta, \frac{\sqrt{\tau}}{\epsilon}\left(k-k_{0}\right)\right)^{-1} D(\zeta, t)^{-1}\right)^{(2)} \\
& =\left(\begin{array}{l}
0 \\
1
\end{array}\right)+\frac{B^{(2)}(\zeta, t)}{\sqrt{\tau}\left(k-k_{0}\right)}+O\left(\tau^{-1+\operatorname{Im} \nu(\zeta)}\right), \quad \tau \rightarrow \infty, \quad \zeta \in \mathcal{I}, \quad\left|k-k_{0}\right|=\epsilon,
\end{aligned}
$$

where $B(\zeta, t)$ is defined by

$$
B(\zeta, t)=-i \epsilon\left(\begin{array}{cc}
0 & -\beta^{X}(\zeta) e^{-t \phi(\zeta, 0)} \tau^{-i \nu(\zeta)} \\
\gamma^{X}(\zeta) e^{t \phi(\zeta, 0)} \tau^{i \nu(\zeta)} & 0
\end{array}\right)
$$

Using (4.47) and 4.50) we find

$$
\begin{aligned}
& \int_{\left|k-k_{0}\right|=\epsilon}\left(\hat{\mu}(\zeta, t, k)\left(m_{0}(\zeta, t, k)^{-1}-I\right)\right)^{(2)} d k=\int_{\left|k-k_{0}\right|=\epsilon}\left(m_{0}(\zeta, t, k)^{-1}-I\right)^{(2)} d k \\
& +\int_{\left|k-k_{0}\right|=\epsilon}(\hat{\mu}(\zeta, t, k)-I)\left(m_{0}(\zeta, t, k)^{-1}-I\right)^{(2)} d k \\
& =\frac{B^{(2)}(\zeta, t)}{\sqrt{\tau}} \int_{\left|k-k_{0}\right|=\epsilon} \frac{d k}{k-k_{0}}+O\left(\epsilon \tau^{-1+\operatorname{Im} \nu(\zeta)}\right)+O\left(\|\hat{\mu}(\zeta, t, \cdot)-I\|_{L^{2}(\hat{\Gamma})} \epsilon^{\frac{1}{2}} \tau^{-\frac{1}{2}+\operatorname{Im} \nu(\zeta)}\right) \\
& =\frac{2 \pi i B^{(2)}(\zeta, t)}{\sqrt{\tau}}+O\left(\epsilon \tau^{-1+\operatorname{Im} \nu(\zeta)}\right)+O\left(\epsilon \tau^{-\frac{1+\alpha}{2}+|\operatorname{Im} \nu(\zeta)|+\operatorname{Im} \nu(\zeta)}\right) \\
& =\frac{2 \pi i B^{(2)}(\zeta, t)}{\sqrt{\tau}}+O\left(\epsilon \tau^{-\frac{1+\alpha}{2}+|\operatorname{Im} \nu(\zeta)|+\operatorname{Im} \nu(\zeta)}\right), \quad \tau \rightarrow \infty, \quad \zeta \in \mathcal{I},
\end{aligned}
$$

uniformly with respect to $\zeta \in \mathcal{I}$. Notice that $B^{(2)}(\zeta, t)$ contains $\tau^{-i \nu(\zeta)}$, the order of the leading term of 4.51 is $\tau^{-\frac{1}{2}+\operatorname{Im} \nu(\zeta)}$. Since $\alpha \in(\lambda, 1)$, the error term of 4.51) does make sense compared to the leading term. 
On the other hand,

$$
\begin{aligned}
\left|\int_{\Gamma}(\hat{\mu}(\zeta, t, k) \hat{w}(\zeta, t, k))^{(2)} d k\right| & =\left|\int_{\Gamma}(\hat{\mu}(\zeta, t, k)-I) \hat{w}^{(2)}(\zeta, t, k) d k+\int_{\Gamma} \hat{w}^{(2)}(\zeta, t, k) d k\right| \\
& \leq\|\hat{\mu}-I\|_{L^{2}(\Gamma)}\left\|\hat{w}^{(2)}\right\|_{L^{2}(\Gamma)}+\left\|\hat{w}^{(2)}\right\|_{L^{1}(\Gamma)} .
\end{aligned}
$$

4.2 and 4.43 implies that $\left\|\hat{w}^{(2)}\right\|_{L^{1}(\Gamma)}=O\left(\epsilon \tau^{-1}+\epsilon \tau^{-\frac{1+\alpha}{2}+\operatorname{Im} \nu(\zeta)}\right)$, and $\left\|\hat{w}^{(2)}\right\|_{L^{2}(\Gamma)}=$ $O\left(\epsilon^{\frac{1}{2}} \tau^{-1}+\epsilon^{\frac{1}{2}} \tau^{-\frac{1}{4}-\frac{\alpha}{2}+\operatorname{Im} \nu(\zeta)}\right)$. Since $\|\hat{\mu}-I\|_{L^{2}(\Gamma)}=O\left(\epsilon^{\frac{1}{2}} \tau^{-\frac{\alpha}{2}+|\operatorname{Im} \nu(\zeta)|}\right)$ by 4.47 and $\alpha \in(\lambda, 1)$, we find that

$$
\begin{aligned}
\left|\int_{\Gamma}(\hat{\mu}(\zeta, t, k) \hat{w}(\zeta, t, k))^{(2)} d k\right| & =O\left(\epsilon \tau^{-1}+\epsilon \tau^{-\frac{1+\alpha}{2}+\operatorname{Im} \nu(\zeta)}+\epsilon \tau^{-\frac{1}{4}-\alpha+|\operatorname{Im} \nu(\zeta)|+\operatorname{Im} \nu(\zeta)}\right) \\
& =O\left(\epsilon \tau^{-\frac{1+\alpha}{2}+|\operatorname{Im} \nu(\zeta)|+\operatorname{Im} \nu(\zeta)}\right), \quad \tau \rightarrow \infty, \quad \zeta \in \mathcal{I},
\end{aligned}
$$

uniformly with respect to $\zeta \in \mathcal{I}$. Then 4.49 (4.50), 4.51) and 4.52 imply (4.44).

Theorem 4.5. Consider the Cauchy problem (1.1). We assume that the scattering data associated the initial data $q_{0}(x)$ are such that:

(1) $a_{1}(k)$ and $a_{2}(k)$ have no zeros in $\overline{\mathbb{C}_{+}}$and $\overline{\mathbb{C}_{-}}$respectively;

(2) For $\zeta \in \mathcal{I}, \Delta(\zeta)=\int_{-\infty}^{k_{0}} d \arg \left(1-r_{1}(s) r_{2}(s)\right) \in(-\pi, \pi)$, where $r_{1}(s)=\frac{b(s)}{a_{1}(s)}$ and $r_{1}(s)=\frac{b(s)}{a_{2}(s)}$.

Then, for any $\alpha \in(\lambda, 1)$ and $N>0$, the solution $q(x, t)$ defined by (2.21) satisfies

$$
\begin{array}{r}
\left.q(x, t)=\frac{4 \epsilon \operatorname{Re} \beta(\zeta, t)}{\tau^{\frac{1}{2}-\operatorname{Im} \nu(\zeta)}+O\left(\epsilon \tau^{-\frac{1+\alpha}{2}}+|\operatorname{Im} \nu(\zeta)|+\operatorname{Im} \nu(\zeta)\right.}\right), \\
\tau \rightarrow \infty,-N t<x<0,
\end{array}
$$

where the error term is uniform with respect to $x$ in the given range.

Proof. Lemma 4.4 implies that 2.21) exists for all sufficiently large $\tau$, and

$$
\begin{aligned}
& q(x, t)=2 i \lim _{k \rightarrow \infty}(k M(x, t, k))_{12}=2 i \lim _{k \rightarrow \infty}(k \hat{m}(x, t, k))_{12} \\
& =\frac{4 \epsilon \operatorname{Re} \beta(\zeta, t)}{\tau^{\frac{1}{2}-\operatorname{Im} \nu(\zeta)}}+O\left(\epsilon \tau^{-\frac{1+\alpha}{2}+|\operatorname{Im} \nu(\zeta)|+\operatorname{Im} \nu(\zeta)}\right) .
\end{aligned}
$$

Remark 4.6. In contrast with the local $m K d V$ equation, the decay rate of the leading term depends on $\zeta=\frac{x}{t}$ through $\operatorname{Im} \nu(\zeta)$. Notice that in the local case, $\operatorname{Im} \nu(\zeta)=0$ for all $\zeta \in \mathcal{I}$, and Theorem 4.5 regresses to the main result of [22].

Remark 4.7. In section 4 of [26], the conditions (1) and (2) in Theorem 4.5 were verified in the case of single box initial data, for which the scattering data can be calculated explicitly. 


\section{ApPendix A.}

Lemma A.1. The $R H$ problem (3.31) has a unique solution $m^{X}(\zeta, z)$ for each $\zeta \in \mathcal{I}$. This solution satisfies

$$
m^{X}(\zeta, z)=I+\frac{i}{z}\left(\begin{array}{cc}
0 & -\beta^{X}(\zeta) \\
\gamma^{X}(\zeta) & 0
\end{array}\right)+O\left(\frac{1}{z^{2}}\right), \quad z \rightarrow \infty, \zeta \in \mathcal{I},
$$

where the error term is uniform with respect to $\arg z \in[0,2 \pi]$ and $\zeta \in \mathcal{I}$. The functions $\beta^{X}(\zeta)$ and $\gamma^{X}(\zeta)$ are defined by

$$
\begin{gathered}
\beta^{X}(\zeta)=\frac{\sqrt{2 \pi} e^{i \pi / 4} e^{-\pi \nu(\zeta) / 2}}{q_{1}(\zeta) \Gamma(-i \nu(\zeta))}, \\
\gamma^{X}(\zeta)=\frac{\sqrt{2 \pi} e^{-i \pi / 4} e^{-\pi \nu(\zeta) / 2}}{q_{2}(\zeta) \Gamma(i \nu(\zeta))} .
\end{gathered}
$$

Moreover, for each closed disk $K \in \mathbb{C}$ centered at the origin,

$$
\sup _{\zeta \in \mathcal{I}} \sup _{z \in K \backslash X}\left|m^{X}(\zeta, z) z^{i \nu(\zeta) \sigma_{3}}\right|<\infty
$$

Proof. The detailed proofs can be found in Appendix A in [26] and Appendix B in [22]. Notice that $m^{X}(\zeta, z)$ is singular at the origin, which is different from the local case. Multiplying $m^{X}(\zeta, z)$ by $z^{i \nu(\zeta) \sigma_{3}}$ can remove the singularity.

\section{REFERENCES}

[1] M. J. Ablowitz and P. A. Clarkson, Solitons, Nonlinear Evolution Equations and Inverse Scattering, London Mathematical Society Lecture Note Series, vol. 149, Cambridge University Press, Cambridge, 1991.

[2] M. J. Ablowitz, D. J. Kaup, A. C. Newell and H. Segur, The Inverse Scattering TransformFourier Analysis for Nonlinear Problems, Studies in Applied Mathematics. 53 (1974), 249-315.

[3] M.J. Ablowitz and Z.H. Musslimani, Integrable nonlocal nonlinear Schrödinger equation, Phys. Rev. Lett. 110064105 (2013).

[4] M. J. Ablowitz and Z. H. Musslimani, Inverse Scattering transform for the integrable nonlocal nonlinear Schrödinger equation, Nonlinearity. 29 (2016), 915-946.

[5] M. J. Ablowitz and Z. H. Musslimani, Integrable nonlocal nonlinear equations, Stud. Appl. Math. 139 (2017), 7-59.

[6] M. J. Ablowitz and H. Segur, Soliton and the Inverse Scattering Transform, SIAM, Philadelphia, 1981.

[7] R. Beals and R. R. Coifman, Scattering and inverse scattering for first order systems, Comm. Pure Appl. Math. 37 (1987), 39-90.

[8] R. Beals, P. A. Deift, and C. Tomet, Direct and Inverse Scattering on the Line, Mathematical Surveys and Monographs, vol. 28, American Mathematical Society, Providence, RI, 1988. 
[9] A. Boutet de Monvel, A. Kostenko, D. Shepelsky, G. Teschl, Long-time asymptotics for the CamassaCHolm equa-tion, SIAM J. Math. Anal. 41 (2009) 1559-1588.

[10] P.J. Cheng, S. Venakides, X. Zhou, Long-time asymptotics for the pure radiation solution of the sine-Gordon equa-tion, Comm. Partial Differential Equations 24 (1999) 1195-1262.

[11] P. Deift, A. R. Its, and X. Zhou, Long-time asymptotics for integrable nonlinear wave equations, Important developments in soliton theory. Springer Ser. Nonlinear Dynam., Springer, Berlin, (1993), 181-204.

[12] P. Deift and X. Zhou, A Steepest Descent Method for Oscillatory Riemann-Hilbert Problems. Asymptotics for the MKdV Equation, Annals of Mathematics. 137 (1993), 295-368.

[13] L. D. Faddeev and L. A. Takhtajan, Hamiltonian Methods in the Theory of Solitons, Reprint of the 1987 English edition, Classics in Mathematics, Springer, Berlin, 2007. Translated from the 1986 Russian original by Al.G. Reyman.

[14] T. A. Gadzhimuradov and A. M. Agalarov, Towards a gauge-equivalent magnetic structure of the nonlocal nonlinear Schrodinger equation, Physical Review A. 93062124 (2016).

[15] K. Grunert, G. Teschl, Long-time asymptotics for the KortewegCde Vries equation via nonlinear steepest descent, Math. Phys. Anal. Geom. 12 (2009) 287

[16] Huang Lin, Lenells Jonatan, Nonlinear Fourier transforms for the sine-Gordon equation in the quarter plane, J. differential equations, 264(2018), 3445-3499

[17] A. R. Its and V. Y. Novokshenov, The Isomonodromic Deformation Method in the Thoery of Painlevé Equation, Lecture Notes in Mathematics, Vol. 1191, Springer, 1986.

[18] J. L. Ji and Z. N. Zhu, On a nonlocal modified Korteweg-de Vries equation: Integrability, Darboux transformation and soliton solutions, Studies in Applied Mathematics. 42 (2017), 699-708.

[19] J. L. Ji and Z. N. Zhu, Soliton solutions of an integrable nonlocal modified Korteweg-de Vries equation through inverse scattering transform, Journal of Mathematical Analysis and Applications. 453 (2017), 973-984.

[20] A.V. Kitaev, A.H. Vartanian, Asymptotics of solutions to the modified nonlinear Schrödinger equation: solution on a nonvanishing continuous background, SIAM J. Math. Anal. 30 (1999) $787-832$.

[21] A.V. Kitaev, A.H. Vartanian, Higher order asymptotics of the modified non-linear Schrödinger equation, Comm. Par-324.tial Differential Equations 25 (2000) 1043-1098.

[22] J. Lenells, The Nonlinear Steepest Descent Method for Riemann-Hilbert Problems of Low Regularity, Indiana University Mathematics Journal. 66 (2017), 1287-1332.

[23] S. Y. Lou and F. Huang, Alice-Bob Physics: Coherent Solutions of Nonlocal KdV Systems, Scientific Reports. 7 (2017), 869. 
[24] K. G. Makris, R. El-Ganainy, D. N. Christodoulides and Z. H. Musslimani, Beam dynamics in PT symmetric optical lattices, Physical Review Letters. 100(2008), 103904.

[25] Z. H. Musslimani, K. G. Makris, R. El-Ganainy and D. N. Christodoulides Optical solitons in PT periodic potentials, Physical Review Letters. 100(2008), 030402.

[26] Y. Rybalko and D. Shepelsky, Long-time asymptotics for the integrable nonlocal nonlinear Schrödinger equation, preprint, available at https://arxiv.org/abs/1710.07961.

[27] X. Y. Tang, Z. F. Liang and X. Z. Hao, Nonlinear waves of a nonlocal modified KdV equation in the atmospheric and oceanic dynamical system, Communications in Nonlinear Science and Numerical Simulation. 60 (2018), 62-71.

[28] J. Xu, Long-time asymptotics for the short pulse equation, J. Differential Equations, accepted

[29] J. Xu and E. G. Fan, Long-time asymptotics for the Fokas-Lenells equation with decaying initial value problem: Without solitons, J. Differential Equations, 259(2015), 1098-1148.

[30] J. Xu and E. G. Fan, Long-time asymptotic behavior for the complex short pulse equation, arXiv:1712.07815.

[31] J. Xu, E. G. Fan and Y. Chen, Long-time Asymptotic for the Derivative Nonlinear Schrodinger Equation with Step-like Initial Value, Math Phys Anal Geom, 16(2013), 253-288.

[32] B. Yang and J. Yang, Transformations between Nonlocal and Local Integrable Equations, Studies in Applied Mathematics. 140 (2017), 178-201.

[33] Q. Z. Zhu, J. Xu and E. G. Fan, The RiemannCHilbert problem and long-time asymptotics for the Kundu-Eckhaus equation with decaying initial value, Applied Mathematics Letters, 76 (2018) 81-89. 